Research Article

\title{
Establishment of an Immune-Related Gene Signature for Risk Stratification for Patients with Glioma
}

\author{
Jimin He $\mathbb{D}^{D}$, Chun Zeng $\mathbb{D}$, and Yong Long $\mathbb{B}$ \\ Department of Neurosurgery, Suining Central Hospital, Suining, China \\ Correspondence should be addressed to Yong Long; 87975481@qq.com
}

Received 30 April 2021; Revised 14 July 2021; Accepted 11 August 2021; Published 28 August 2021

Academic Editor: Maria N. D.S. Cordeiro

Copyright (c) 2021 Jimin He et al. This is an open access article distributed under the Creative Commons Attribution License, which permits unrestricted use, distribution, and reproduction in any medium, provided the original work is properly cited.

\begin{abstract}
Glioma is a frequently seen primary malignant intracranial tumor, characterized by poor prognosis. The study is aimed at constructing a prognostic model for risk stratification in patients suffering from glioma. Weighted gene coexpression network analysis (WGCNA), integrated transcriptome analysis, and combining immune-related genes (IRGs) were used to identify core differentially expressed IRGs (DE IRGs). Subsequently, univariate and multivariate Cox regression analyses were utilized to establish an immune-related risk score (IRRS) model for risk stratification for glioma patients. Furthermore, a nomogram was developed for predicting glioma patients' overall survival (OS). The turquoise module (cor $=0.67 ; P<0.001)$ and its genes $(n=1092)$ were significantly pertinent to glioma progression. Ultimately, multivariate Cox regression analysis constructed an IRRS model based on VEGFA, SOCS3, SPP1, and TGFB2 core DE IRGs, with a C-index of 0.811 (95\% CI: 0.786-0.836). Then, Kaplan-Meier $(\mathrm{KM})$ survival curves revealed that patients presenting high risk had a dismal outcome $(P<0.0001)$. Also, this IRRS model was found to be an independent prognostic indicator of gliomas' survival prediction, with $\mathrm{HR}$ of 1.89 (95\% CI: 1.252-2.85) and 2.17 (95\% CI: 1.493-3.14) in the Cancer Genome Atlas (TCGA) and Chinese Glioma Genome Atlas (CGGA) datasets, respectively. We established the IRRS prognostic model, capable of effectively stratifying glioma population, convenient for decision-making in clinical practice.
\end{abstract}

\section{Introduction}

Glioma is a common primary malignant brain tumor [1], with the average incidence of approximately six cases per 100,000 people in the world [2]. According to the WHO classification, glioma can be divided into four grades, including grades I, II, III, and IV, where grades I and II are lowgrade glioma (LGG), including astrocytoma, oligodendroglioma, and mixed oligodendrogliomas, whereas grades III and IV are high-grade gliomas (HGG), such as glioblastoma (GBM), anaplastic astrocytoma, and anaplastic oligodendroglioma [3]. However, as time goes by, LGG will progress to invasive HGG [4-6]. On the basis of the statistical data of the Chinese Glioma Genome Atlas (CGGA), the overall survival (OS) of LGG is 78.1 months, with a 5 -year survival rate of $67 \%$ [7] . Nevertheless, once patients progress to HGG, their OS will shorten to 14.4 months and the 5-year survival rate is roughly $9 \%$. Even though they adopt positive treat- ments, such as surgery and chemotherapy, as well as radiotherapy, their 2-year survival rate is merely $43 \%$ [8].

Immunotherapeutic strategies in glioma arouse unprecedented attention in the public, where long-term tumor remission can be achieved with minimal side-effects [9]. The immune therapeutic methods included immune checkpoint inhibitors (ICI), peptide vaccines, dendritic cell vaccines, chimeric antigen receptor $\mathrm{T}$ cells, and oncolytic viruses [10]. The ICIs contain the programmed cell death protein (PD-1) and its ligand (PD-L1), considered as the main factors hindering immune response [11]. However, due to low and variable levels of PD-L1 in glioma cells [12, 13], most patients showed no significant increase in OS for anti-PD-1 therapy [14]. Thus, it is imperative to develop some potential consistently expressed immune-related biomarkers of the glioma. In recent years, some researchers propose that immune-related gene (IRG) signatures are involved in tumor prognosis, including pancreatic ductal 
adenocarcinoma [15], lung adenocarcinoma [16], neuroblastoma [17], and head and neck squamous cell carcinoma [18], as well as LGG [19]. In a previous study, Wang et al. have identified an immune-related lncRNA signature via construction of immune-lncRNAs and an immune-gene coexpression network, for predicting its prognostic value in the anaplastic gliomas [20]. Moreover, another previous study has established an immune-related risk signature for GBM, which can independently distinguish high-risk patients, and they also elucidated the relationship between local immune response and prognosis in GBM [21]. In addition, Zhang et al. constructed an IRG signature for risk stratification and developed a prognostic nomogram for survival prediction in LGG patients [19]. However, most researches established prognostic models based on IRG signatures [19, 21-23], but they have yet to illuminate whether progression-related differentially expressed IRGs (DE IRGs) can effectively perform risk stratification for patients subjected to glioma.

Hence, this present study was undertaken to develop a prognostic model via screening progression-related core DE IRGs [24], for stratifying glioma patients by risk score and providing potential immunotherapeutic targets for inhibition of glioma progression and improvement of patients' prognosis.

\section{Materials and Methods}

2.1. Data Downloading and Processing. By searching the GEO database (http://www.ncbi.nlm.nih.gov/geo/), original data (CEL format) of GSE4290 were downloaded and decompressed to perform weighted gene coexpression network analysis (WGCNA). The LGG and HGG samples from GSE45921 [25], GSE15824 [26], GSE43378 [27], and GSE4290 [28] datasets were also selected for differential expression analysis, all of which have same annotation platform, namely, GPL570 (HG-U133_Plus_2, Affymetrix Human Genome U133 Plus 2.0 Array), with 54,675 probes. Besides, transcriptome and clinical data (including sample id, age, gender, status, survival time, tumor grade, isocitrate dehydrogenase $(I D H)$ status, and O-6-methylguanine-DNA methyltransferase (MGMT) status) of all glioma samples from the TCGA-LGG (level 3) and TCGA-GBM (level 3) were downloaded from the TCGA database (https://portal .gdc.cancer.gov/) and cBioPortal for Cancer Genomics database (http://www.cbioportal.org/), respectively. Next, we attained a total of 2498 IRGs from the Immunology Database and Analysis Portal (ImmPort) (https://www.immport .org/shared/home) [18, 29]. Subsequently, we collected RNA-seq data and corresponding clinical information from the CGGA database (http://www.cgga.org.cn/) [30, 31] for further verification.

2.2. Weighted Gene Coexpression Network Analysis. Genes with top-ranking 5000 expression data of the GSE4290 dataset were used to implement WGCNA. Then, coexpression modules were constructed using the "WGCNA" package in the RStudio software (Version 3.5.0). To eliminate outliers, the parameters of height and minsize were set to 110 and
30, respectively. Using the power function $a_{\mathrm{mn}}=\left|c_{\mathrm{mn}}\right|^{\beta}$ $\left(a_{\mathrm{mn}}\right.$ : adjacency between gene $\mathrm{m}$ and gene $\mathrm{n}, c_{\mathrm{mn}}$ : Pearson correlation, $\beta$ : the soft threshold), a weighted adjacency matrix was constructed $[32,33]$. Then, the topological overlap matrix (TOM) was converted to the adjacency matrix to measure the network connectivity of these genes [32]. Subsequently, genes with absolute high correlation were classified into gene modules according to the TOM-based dissimilarity metric [33]. In addition, coexpression modules were uncovered using the blockwiseModules function of the "WGCNA" package [34, 35]. Through establishment of a hierarchical clustering tree diagram of selected gene expression values and analysis of adaptive branch cutting [36], functional modules were visualized with different colors [37], where genes without being enriched in any module were assigned to the gray module [38]. Module eigengene (ME) is defined as the main component of the first standardized expression profile [39] and also is thought as the representative gene expression profile in modules [33, 40]. Furthermore, module significance tends to be highly correlated with the correlations between $\mathrm{ME}$ and clinical traits [41]. And gene significance (GS) is considered to be the absolute values of the correlation coefficients between genes and traits, and module membership (MM) is defined as the correlations between the eigengene modules and gene expression profile [34, 42]. Accordingly, a preliminary correlation between modules and clinical traits was revealed via the WGCNA method.

2.3. Identification of Differentially Expressed Genes (DEGs) and Their IRGs (DE IRGs). The original data from four GEO datasets (including GSE45921, GSE15824, GSE43378 and GSE4290) were decompressed and implemented normalization, background correction, and $\log 2$ transformation via the "affy" package $[43,44]$. And missing values were filled using the impute.knn function [45]. Additionally, the ComBat algorithm in the "sva" package was adopted for removing batch-batch difference [46]. Subsequently, differential expression analysis between HGG and LGG samples was performed using the "limma" package. If multiple probes matched to the same gene symbol, the median expression values were selected as the expression levels of these genes. Besides, cutoff values of DEGs were set to । $\log _{2}$ FoldChange $\left(\log _{2}\right.$ FC) $\mid \geq 1.0$ and adjusted $P<0.05$. To further discover glioma progression-related DE IRGs, a Venn diagram was used based on an overlapping region of DEGs and IRGs, as well as genes in the significant progression-related modules.

2.4. Functional Enrichment Analyses for DE IRGs. The Gene Ontology (GO) term and Kyoto Encyclopedia of Genes and Genomes (KEGG) functional enrichment analyses were performed for DE IRGs via the "ClusterProfiler" [47] package. GO term enrichment analysis contained three categories, namely, molecular function (MF), cellular component (CC), and biological processes (BP) [48]. Additionally, KEGG (http://www.genome.jp/kegg/) is a bioinformatics resource that links genome or molecular datasets to 
networks [49]. The $P$ value less than 0.05 was regarded as the threshold.

2.5. Construction of a PPI Network and Identification of Clusters. A protein-protein interaction (PPI) network of DE IRGs was constructed using the STRING (https:// string-db.org/) [50] website via setting the minimum required interaction score to 0.900 and the max number of interactors to show to no more than 50 , and visualized in the Cytoscape software [51]. Furthermore, the median degree was calculated by the CentiScaPe 2.0 plug-in, a tool for determining the most significant nodes [52]. And the molecular complex detection (MCODE) [53] plug-in was capable of visualizing the highly connected clusters of the PPI network. Thus, significant nodes were selected as core genes for the next analysis. A GOChord function in the "GOplot" package was employed to display the relationships that genes were coupled with GO terms through ribbons [54].

2.6. Survival Analysis. To disclose the predictive value of expression levels of DE IRGs for glioma survival, we adopted a log rank test to perform Kaplan-Meier (KM) survival analyses.

2.7. Establishment of an Immune-Related Risk Score (IRRS) Model. We screened out the most significant nodes via the previous methods. The TCGA samples were used to construct and assess the IRRS model. Firstly, univariate Cox regression analysis was performed to screen those genes with $P<0.05$. Subsequently, the IRRS model was established by multivariate Cox regression analysis based on the Akaike Information Criterion (AIC) algorithm [55]. The risk score formula was erected via the coefficients $(\beta)$ of each gene from the multivariate Cox regression analysis and their expression levels. The calculation formula of the risk score was as follows: risk score $=$ expression level of $\mathrm{mRNA} 1 \times \beta$ $1_{\text {mRNA1 }}+$ expression level of mRNA $2 \times \beta 2_{\text {mRNA2 }}+\cdots+$ expression level of $\mathrm{mRNAn} \times \beta n_{\mathrm{mRNAn}}$. Then, the timedependent receiver operating characteristic (ROC) curves for predicting the 1-year, 3-year, and 5-year OS showed the predictive performance of this IRRS model via the "survivalROC" package [56]. In addition, the concordance index (C-index), ranging from 0.5 to 1.0 , is an indicator for evaluating this model's predictive ability. The closer to 1.0 the Cindex, the better the discriminatory ability of the IRRS model [57]. Next, considering that 5 years is a key time point for evaluating the prognosis of glioma patients, we have stratified patients into high- and low-risk groups via adopting the optimal cutoff value of the risk score at the 5-year time point in ROC curves [58]. Subsequently, survival analysis was implemented using the "survival" and "survminer" packages via log rank test, and the 5-year survival rate also was acquired.

2.8. Evaluation of the Independent Prognostic Value of the IRRS Model. We have performed univariate and multivariate Cox regression analyses for the IRRS model and clinical characteristics, that is, a combination of age $(<60$ and $\geq 60$ ), gender (male and female), tumor grade (low grade and high grade), risk score (low risk and high risk), IDH status (mutant and wild-type), and MGMT status (methylated and unmethylated). Subsequently, the nomogram with 1year, 3-year, and 5-year OS rates was developed for clinical use [59]. Moreover, calibration curves were utilized to evaluate the agreement of predictive and observed probabilities of 1-year, 3-year, and 5-year OS from the nomogram.

2.9. Statistical Analysis. Statistical analysis was conducted in the RStudio software (Version 3.5.0) and GraphPad Prism software (Version 5.0). Survival curves were established via the KM method and log-rank test. Calculation of the area under ROC curves of 1-year, 3-year, and 5-year survival rates was implemented based on the "survivalROC" package [56]. The "rms" package was applied to perform nomogram analysis. $P<0.05$ denoted the presence of statistically significant difference.

\section{Results}

3.1. Data Processing. The flowchart of the present study design is presented in Figure 1. First, we selected the topranking 5000 gene expression values of the GSE4290 dataset based on the median absolute derivation (MAD) algorithm for performing WGCNA. Second, integrated transcriptome analysis was conducted in a total of 251 samples, including 179 HGG and 72 LGG samples, derived from four GEO datasets, among which 7 HGG and 15 LGG in the GSE45921 dataset, 19 HGG and 7 LGG in the GSE15824 dataset, 45 HGG and 5 LGG in the GSE43378 dataset, and 108 HGG and 45 LGG in the GSE4290 dataset. In addition, 687 duplicate IRGs were removed from IRG group. Then, fragments per kilobase per million normalized expressions of RNA-seq data (level 3) of 529 LGG and 169 GBM samples in the TCGA database were used for construction of the prognostic model. Due to 10 samples lacking information of survival time and status, we ultimately used 688 glioma samples to construct the prognostic model and validate its predictive performance. Additionally, 422 primary glioma patients from the CGGA database were selected as the external validation set.

\subsection{Construction of a Weight Gene Coexpression Network} and Identification of Grade Progression-Related Modules. First, we used a hierarchical clustering method to detect the outliers, enabling two samples to be eliminated (“GSM97895" and "GSM97932"). The soft threshold value was equal to 12 via the pickSoftThreshold function, shown in Figure 2(a). Second, we employed the blockwiseModules function of the "WGCNA" package to acquire six modules, including brown, turquoise, green, blue, yellow and gray modules, with genes ranging from 149 to 1552, where the gray module represented genes without being enriched, which is visualized in Figure 2(b). Third, we analyzed the relationship between samples and clinical traits (such as grade and subtype of glioma) and obtained the correlation of modules with traits. And Figure 2(c) showed that the turquoise module was significantly positively associated with glioma grade (cor $=0.67, P<0.0001)$. Then, a scatter plot 


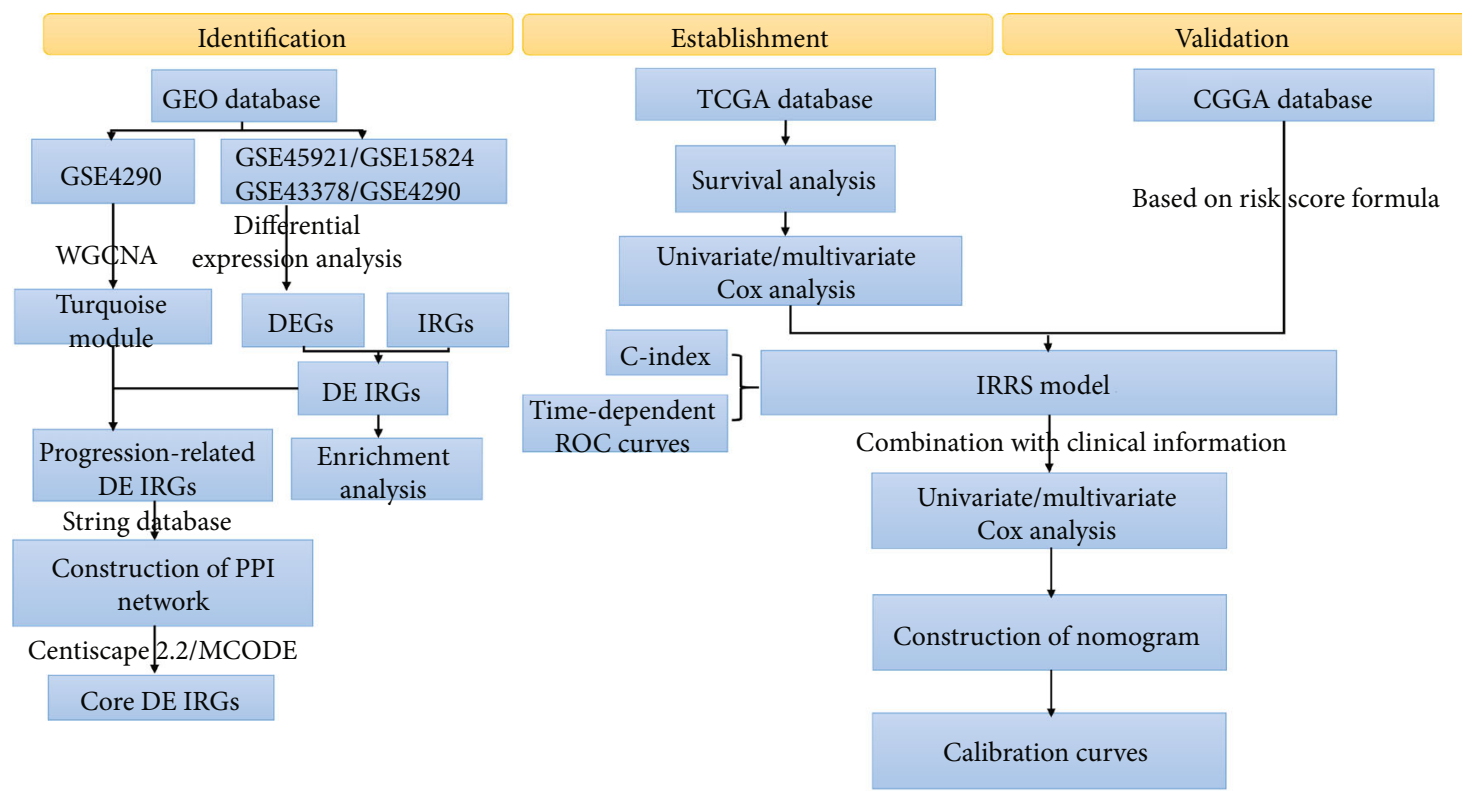

Figure 1: The flow chart of the present study.

of MM vs. GS in the turquoise module was drawn, with a correlation coefficient of $0.76(P<0.0001)$ (Figure $2(\mathrm{~d}))$. Additionally, 1092 genes in the turquoise module were identified (Supplementary Table 1).

3.3. Differentially Expressed Genes (DEGs) and Their IRGs (DE IRGs), as well as Functional Enrichment Analyses. Differential expression analysis between HGG $(n=179)$ and LGG $(n=72)$ samples was completed via the "limma" package. According to the presetting cutoff value, 389 DEGs (Supplementary Table 2) were obtained for further analysis, with upregulation of 213 and downregulation of 176 DEGs. Figure 3(a) shows a clustering heat map that reveals the expression levels of five core DE IRGs. We selected the 41 overlapping genes in the DEG and IRG groups via Venn plot as DE IRGs. Thus, as shown in Figure 3(b), 26 progression-related DE IRGs located in the intersection region among the DEG, IRG, and turquoise module's gene groups. Subsequently, Figures 3(c) and 3(d) present the results of GO term and KEGG pathway enrichment analyses, respectively, which clarified that these DE IRGs were mainly enriched in receptor ligand activity, growth factor activity, extracellular matrix binding, etc. (GO term), as well as focal adhesion (KEGG pathway).

3.4. Construction of a PPI Network and Identification of Clusters. A PPI network of 26 progression-related DE IRGs was constructed by the STRING tool and was visualized via the Cytoscape software, with 118 nodes and 705 edges (Figure 4(a)). The median degree, as the threshold of PPI network, was 11.94 calculated by the CentiScaPe 2.2 plugin. Subsequently, a total of four closely connected clusters were discovered (Table 1), with a score more than 6.0 using the MCODE algorithm, where the parameters of degree cutoff, node score cutoff, $k$-score, and max. depth were set to $2.0,0.2,2.0$, and 100, respectively (Figures $4(\mathrm{~b})-4(\mathrm{e})$ ). Ulti- mately, we have identified five core DE IRGs (including VEGFA, SOCS3, THBS1, SPP1, and TGFB2 genes) with a degree more than 12 in clusters $A$ and $B$, which met the requirements mentioned above. Additionally, GOChord diagrams disclosed that most of core DE IRGs were enriched in the positive regulation of epithelial cell migration (BP, Figure 4(f)) and extracellular matrix binding and cytokine activity, as well as receptor ligand activity (MF, Figure $4(\mathrm{~g})$ ).

3.5. Evaluation of the Impact of Core DE IRGs on Glioma Prognosis. Survival curves of five core DE IRGs indicated that high levels of core DE IRGs were associated with shorter OS of glioma patients (Table 2, Figure 5, $P<0.0001$ ). Additionally, the 5-year survival rates of patients with overexpressed VEGFA, SOCS3, THBS1, SPP1, and TGFB2 genes in $\mathrm{KM}$ survival curves separately were $20.38 \%, 23.85 \%$, $29.61 \%, 24.27 \%$, and $21.93 \%$ based on the TCGA cohort, whereas in the CGGA dataset, for patients with high expression levels of VEGFA, SOCS3, THBS1, SPP1, and TGFB2 genes, the 5-year survival rates were $33.70 \%, 30.20 \%$, $36.70 \%, 38.30 \%$, and $37.60 \%$, respectively (Table 2).

3.6. Establishment of the IRRS Model. These core DE IRGs were used to perform univariate Cox regression analysis, screening genes with a $P$ value less than 0.05 , which were included for conducting multivariate Cox regression analysis via the AIC algorithm (the smallest value of 2753.8) for establishment of a prognostic model. Finally, we constructed an IRRS model composed of four DE IRGs via the following formula: risk score $=0.20365 \times V E G F A+0.12448 \times$ SOCS3 $+0.26231 \times S P P 1+0.12930 \times T G F B 2$. And C-index was 0.811 (95\% CI: 0.786-0.836) for the IRRS model, showing the superiority of this model for predicting the probability of OS in glioma population (Table 3). Subsequently, we used diagrams of survival status' distribution and risk score of each glioma patient, as well as four gene expression heat 

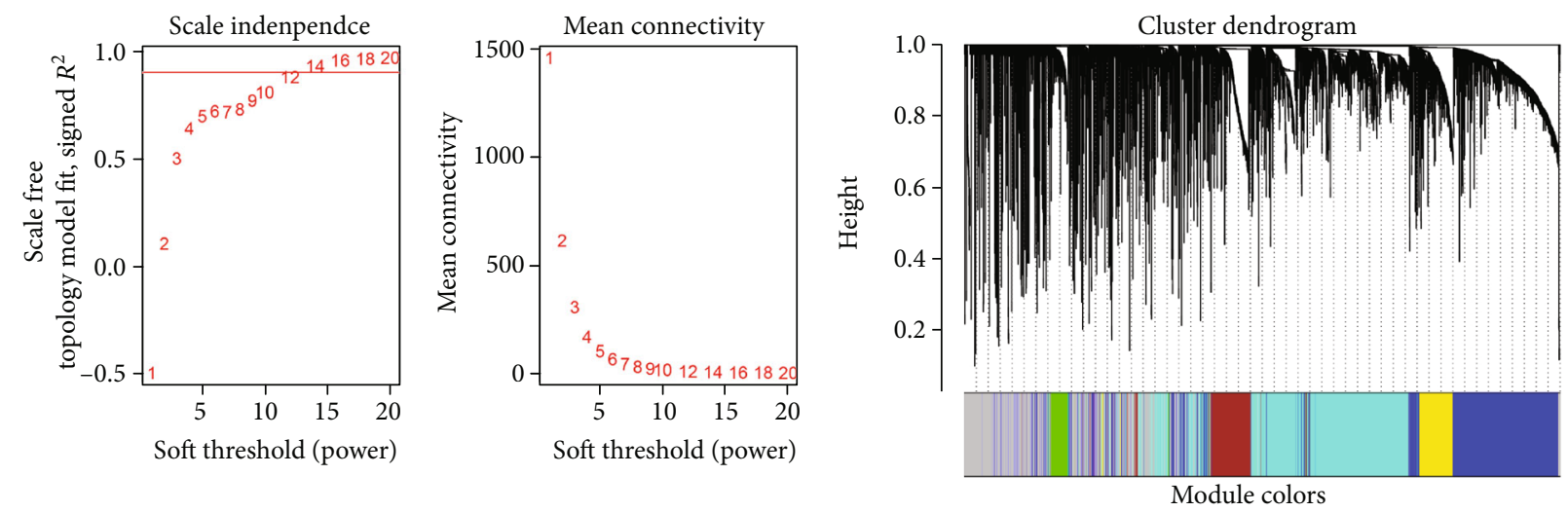

(a)

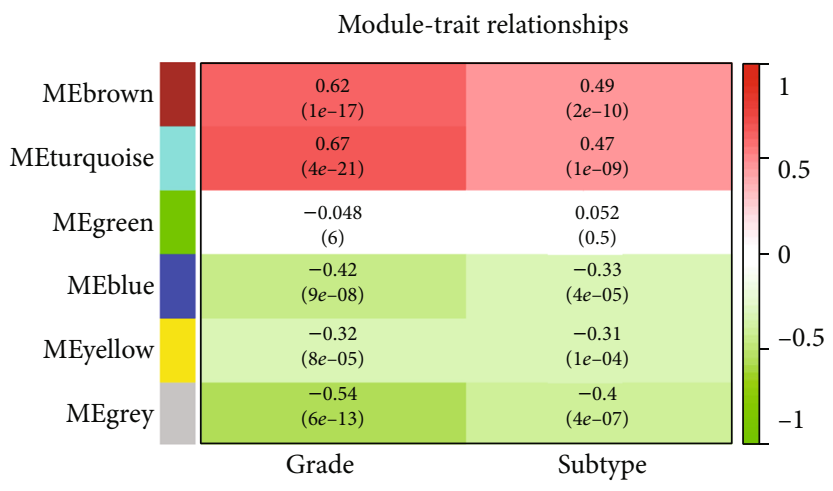

(c)

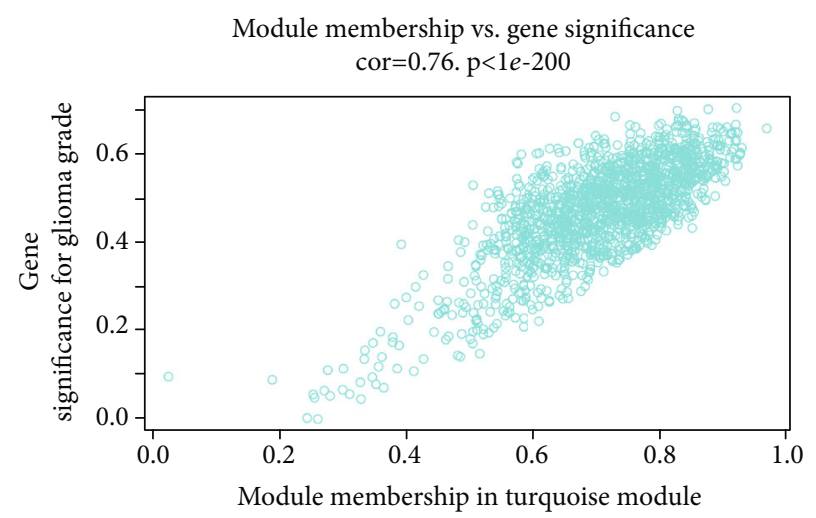

(d)

FIGURE 2: Weighted gene coexpression network analysis. (a) Identification of the soft threshold power on the basis of the criteria of the scalefree network. The left panel shows the relationship between the scale-free fit index ( $y$-axis) and soft threshold power ( $x$-axis). And the right panel shows the impact of soft threshold power ( $x$-axis) on the mean connectivity (degree, $y$-axis). (b) Cluster dendrogram of gene coexpressed modules. A gene clustering dendrogram is plotted using the dissimilarity measure (1-TOM). The colored horizontal bars below the clustering dendrogram represent the modules labeled with different colors, where the gray module shows no genes enriched in any module. (c) The heat map of the correlations between module eigengenes and clinical features of glioma. Each column represents a clinical trait (including grade and subtype) and each row represents a module. Each cell contains the corresponding correlation coefficient on the first row and $P$ value on the second row. The red and green colors show the positive and negative correlations, respectively. And the darker the color of the cell, the stronger the correlation between modules and clinical traits. (d) Scatter plot of the correlation between gene significance for the glioma grade and module membership in the turquoise module $($ cor $=0.76$; $P<0.0001)$. TOM: topological overlap matrix.

maps to illuminate the IRRS model's role in glioma patients (Figure 6(a)). Further evidence implied that the areas under ROC curves for evaluating 1-year, 3-year, and 5-year OS were $0.86,0.855$, and 0.812 , respectively (Figure $6(\mathrm{~b}))$. The optimal cutoff value of the risk score for forecasting the 5year survival in ROC curves was 0.87894 , which was used to classify glioma patients into high- and low-risk populations and then generate a survival curve, revealing that the 5 -year survival rate was merely $14.30 \%$ in the high-risk group (Figure 6(c)). As is shown in Table 4, the correlations between the risk score and clinicopathological characteristics were described. Also, univariate and multivariate Cox regression analyses based on the risk score and clinical characteristics indicated that this IRRS model was able to be an independent prognostic factor (Figure 6(d)), with an HR value of 1.89 (95\% CI: $1.252-2.85, P=0.0024$ ) (Table 5). Due to the trait of gender showing no statistical difference in univariate Cox regression analysis $(\mathrm{HR}=1.26,95 \% \mathrm{CI}$ :
0.986-1.61, $P=0.0645$ ), we excluded the factor of gender; then, we included the rest of the indicators to perform multivariate Cox regression analysis. Furthermore, gliomas' grade was found to be a risk factor of glioma patients (HR $=2.54,95 \%$ CI: $1.750-3.69, P<0.001)$. Also, IDH wild-type also was characterized by an independent prognostic value for glioma patients ( $\mathrm{HR}=2.70,95 \% \mathrm{CI}$ : 1.700 4.28, $P<0.001)$.

3.7. Validation of the IRRS Model. The CGGA cohort was selected as an external validation set. The survival status of each patient, their risk scores' distribution, and the heat map of four genes are shown in Figure 7(a). Similarly, the areas under the curve (AUC) of the risk score at 1-year, 3year, and 5-year time points of ROC curves were 0.715, 0.772 , and 0.759 , respectively (Figure $7(\mathrm{~b})$ ). Also, the optimal cutoff value of 1.27 , calculated by the "survivalROC" package, divided patients into high- and low-risk groups; 


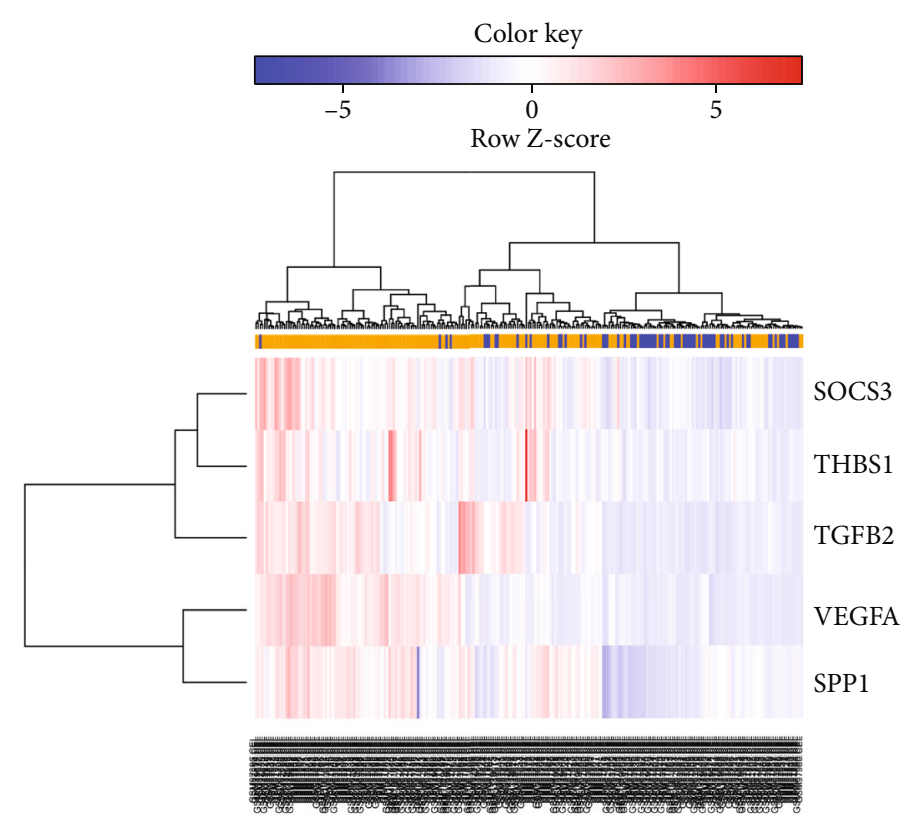

(a)

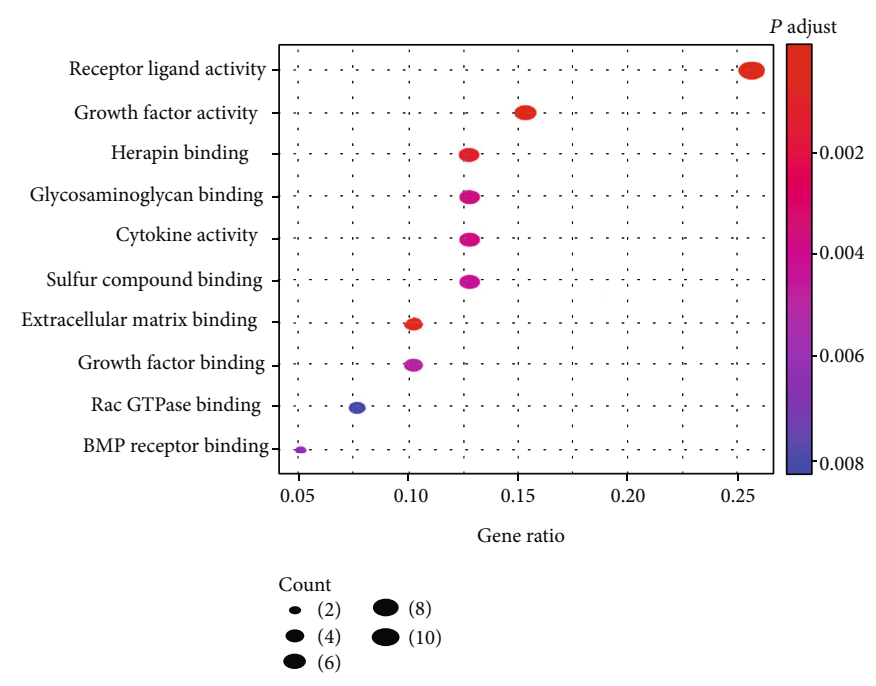

(c)
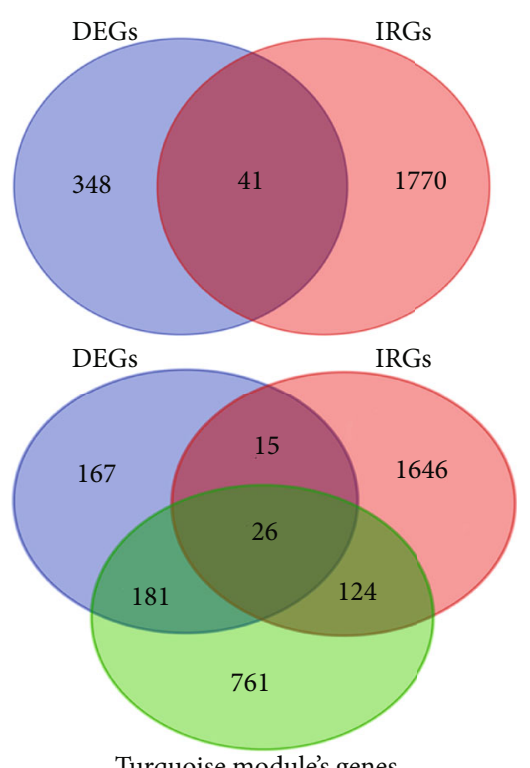

(b)

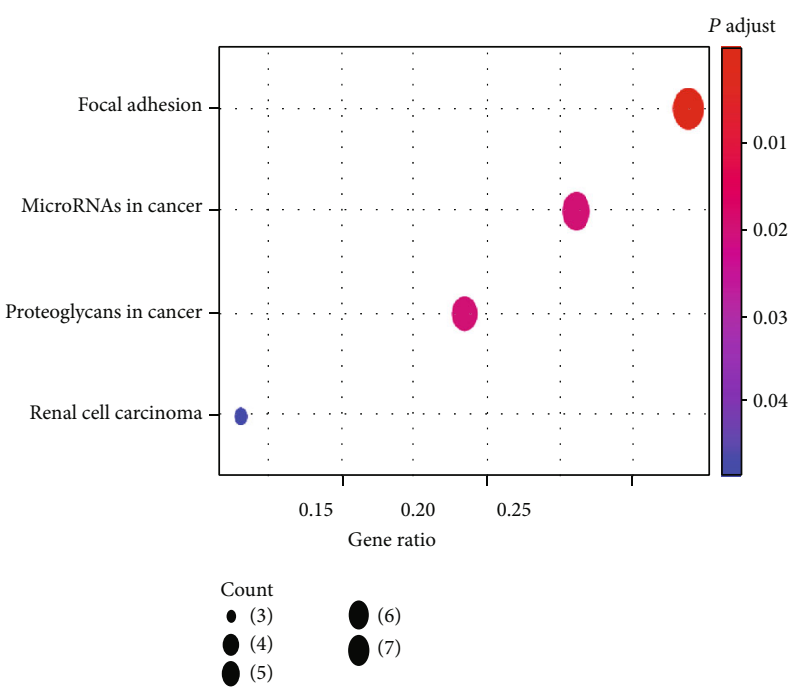

(d)

FIGURE 3: Identification of DE IRGs and functional enrichment analyses. (a) The clustering heat map of five DE IRGs. The row and column represent genes and samples, respectively. (b) Venn plots reveal 41 DE IRGs and 26 progression-related DE IRGs. (c) GO term enrichment analysis for DE IRGs. (d) KEGG pathway enrichment analysis for DE IRGs. DEGs: differentially expressed genes; IRGs: immune-related genes; DE IRGs: differentially expressed immune-related genes; SOCS3: suppressor of cytokine signaling 3; THBS1: thrombospondin 1; TGFB2: transforming growth factor beta 2; VEGFA: vascular endothelial growth factor A; SPP1: secreted phosphoprotein 1; GO: Gene Ontology; KEGG: Kyoto Encyclopedia of Genes and Genomes.

then, the survival curve elucidated that the 5-year survival rate was merely $29.80 \%$ in high-risk glioma patients (Figure 7(c)). Meanwhile, the IRRS model was demonstrated that it can become an independent prognostic indicator (HR: 2.17, 95\% CI: 1.493-3.14, $P<0.0001$, Figure 7(d)).

3.8. Development of a Nomogram. A nomogram was erected to predict 1-year, 3-year, and 5-year OS of glioma patients via combing the IRRS prognostic model and significant clinical characteristics (Figure 8(a)), which appeared to be conducive to clinical use. Furthermore, calibration curves substantiated a superior consistency between predicted and observed values at probabilities of 1-year, 3-year, and 5year OS of the nomogram, as described in Figure 8(b).

3.9. Comparison with Other Prognostic Models of Glioma. Via searching related literature about glioma's prognostic models, we compared these models with this IRRS model (Table 6). Our model had moderate accuracy with AUC of 1 year, 3 years, and 5 years more than 0.70 both in the TCGA and CGGA databases. Despite the presence of higher accuracy in other models, their models were composed of 


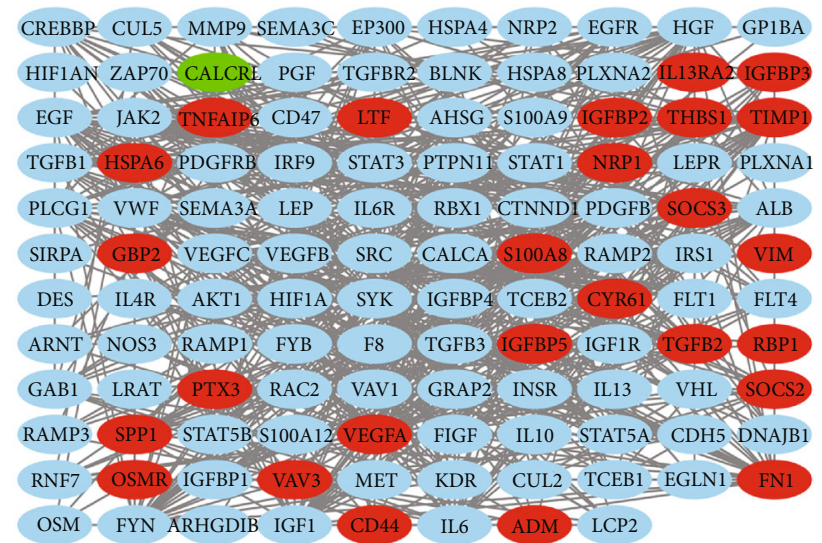

(a)

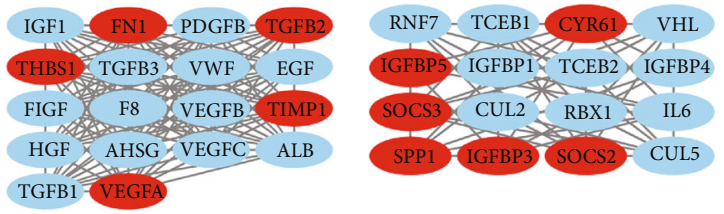

(b)

(c)

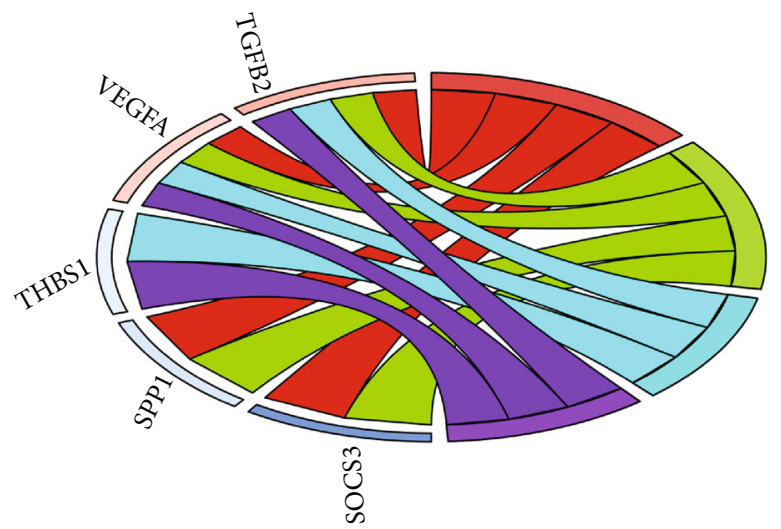

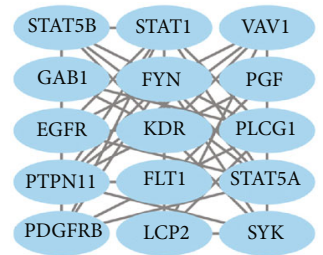

(d)

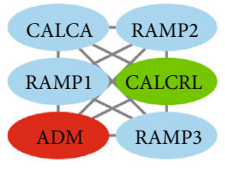

(e)

Reproductive structure development

$\square$ Reproductive system development

$\square$ Platelet degranulation

$\square$ Positive regulation of epithelial cell migration

(f)

Figure 4: Continued. 


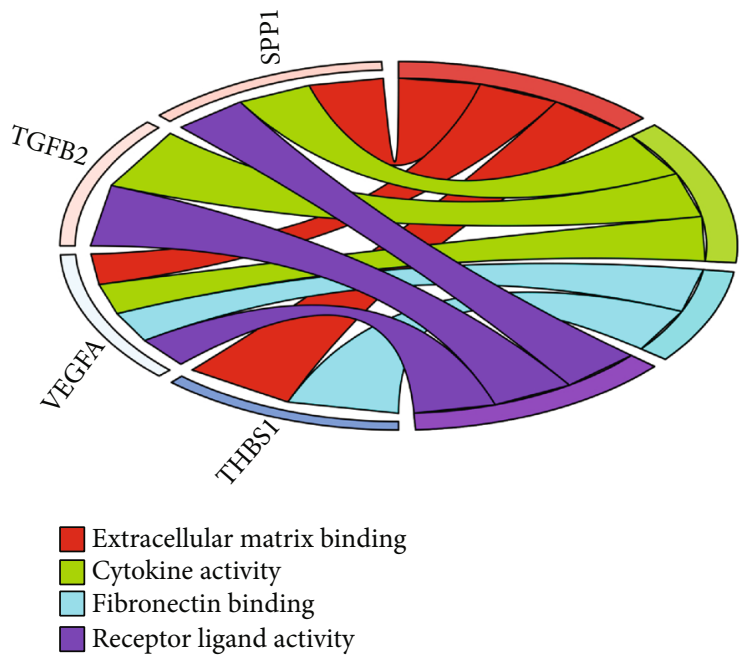

(g)

FIGURE 4: Construction of a PPI network, identification of clusters, and GOChord plot for core DE IRGs. (a) PPI network with 118 nodes and 705 edges, with the red and green representations of upregulated and downregulated DEGs, respectively. (b) Cluster A, (c) cluster B, (d) cluster C, and (e) cluster D. GOChord plot for five core DE IRGs in the (f) "BP" category and (g) "MF" category. PPI: protein-protein interaction; DEGs: differentially expressed genes; DE IRGs: differentially expressed immune-related genes; BP: biological process; MF: molecular function.

TABLE 1: Clusters of PPI network using the MCODE algorithm.

\begin{tabular}{lcccc}
\hline Cluster & Nodes & Edges & Score & Gene symbol \\
\hline A & 18 & 153 & 18 & F8, FIGF, TGFB1, VEGFB, TIMP1, EGF, TGFB2, TGFB3, IGF1, VEGFC, HGF, THBS1, ALB, FN1, AHSG, \\
VEGFA, PDGFB, and VWF
\end{tabular}

PPI: protein-protein interaction; MCODE: molecular complex detection; bold texts indicate differentially expressed genes in the PPI network.

TABLE 2: The hazard ratio of the five DE IRGs for evaluating the impact on gliomas' survival.

\begin{tabular}{|c|c|c|c|c|c|c|c|c|}
\hline \multirow{3}{*}{ Genes } & \multicolumn{4}{|c|}{ TCGA cohort } & \multicolumn{4}{|c|}{ CGGA cohort } \\
\hline & \multirow{2}{*}{ HR (95\% CI) } & \multicolumn{3}{|c|}{ 5-year survival rates (\%) } & \multirow{2}{*}{ HR (95\% CI) } & \multicolumn{3}{|c|}{ 5-year survival rates $(\%)$} \\
\hline & & Low & High & $P$ value & & Low & High & $P$ value \\
\hline VEGFA & $4.03(3.16-5.15)$ & 67.20 & 20.38 & $<0.0001$ & $2.62(1.99-3.45)$ & 67.40 & 33.70 & $2.383 E-12$ \\
\hline SOCS3 & $4.26(3.35-5.43)$ & 65.60 & 23.85 & $<0.0001$ & $3.70(2.80-4.89)$ & 71.40 & 30.20 & $<0.0001$ \\
\hline THBS1 & $2.93(2.31-3.72)$ & 59.80 & 29.61 & $<0.0001$ & $2.25(1.71-2.97)$ & 64.70 & 36.70 & $3.727 E-09$ \\
\hline SPP1 & $3.80(2.98-4.85)$ & 65.50 & 24.27 & $<0.0001$ & $2.21(1.68-2.90)$ & 63.50 & 38.30 & $1.421 E-08$ \\
\hline TGFB2 & $4.64(3.64-5.92)$ & 67.60 & 21.93 & $<0.0001$ & $2.26(1.72-2.97)$ & 65.00 & 37.60 & $6.757 E-09$ \\
\hline
\end{tabular}

TCGA: The Cancer Genome Atlas; CGGA: Chinese Glioma Genome Atlas; HR: hazard ratio; VEGFA: vascular endothelial growth factor A; SOCS3: suppressor of cytokine signaling 3; THBS1: thrombospondin 1; SPP1: secreted phosphoprotein 1; TGFB2: transforming growth factor beta 2; bold texts indicate statistically significant difference. 

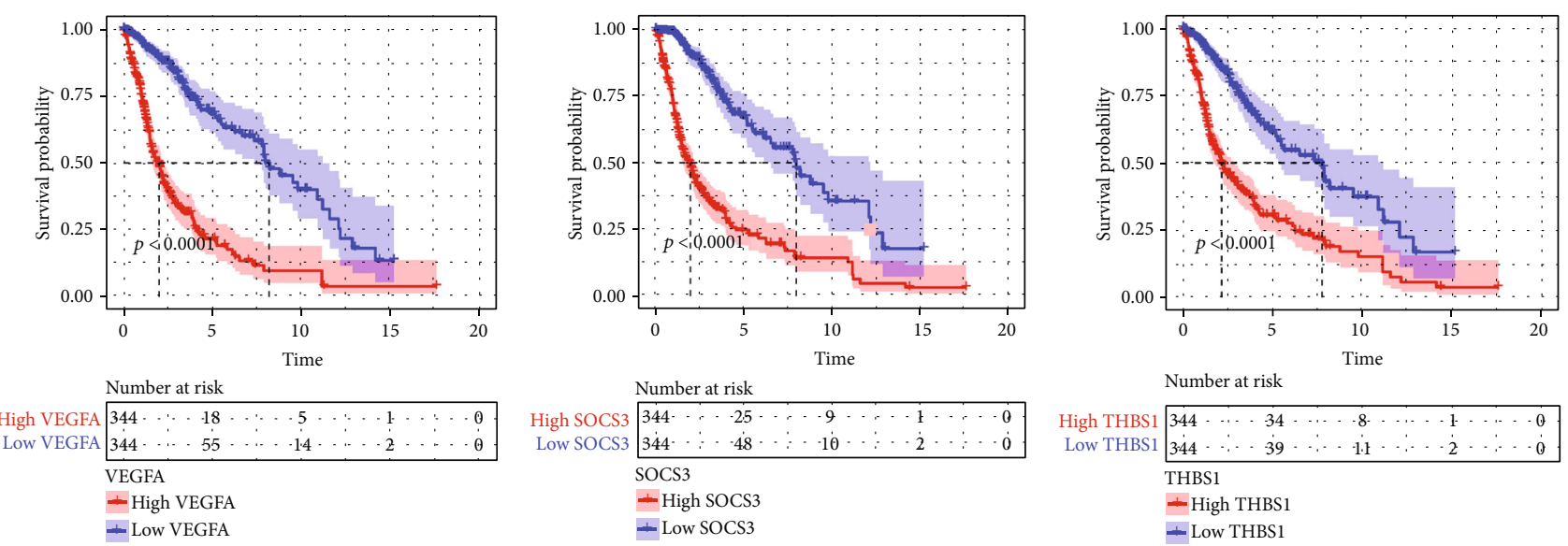

(a)

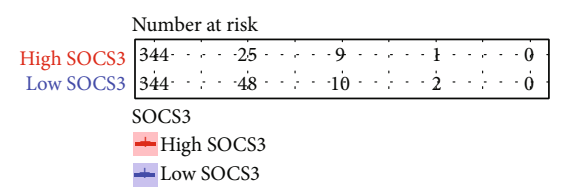

(b)

(c)

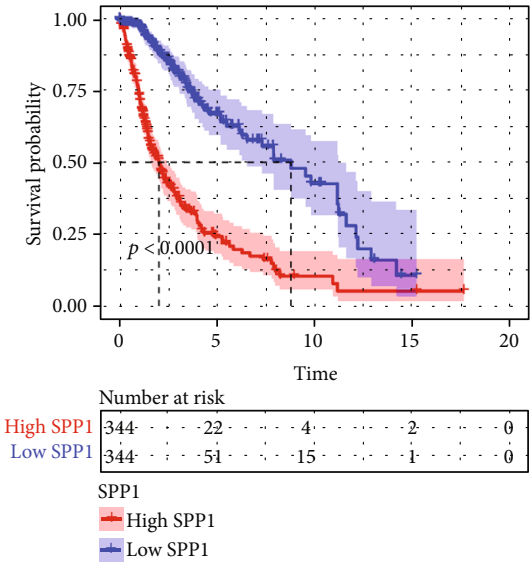

(d)

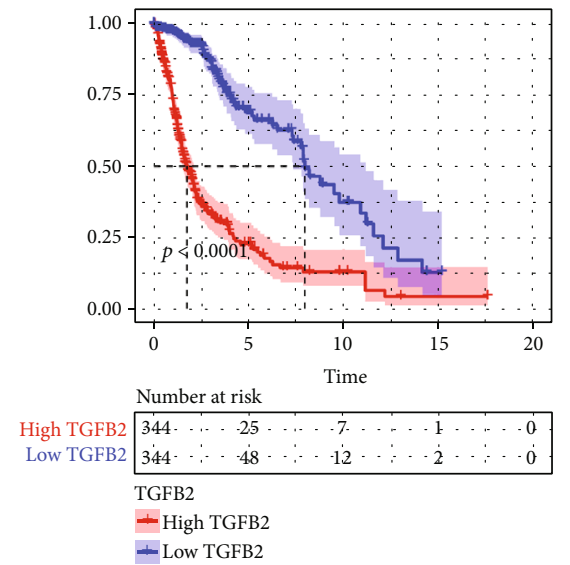

(e)

FIGURE 5: Survival curves uncover the relationships between five core DE IRGs and glioma patients' survival in the TCGA cohort: (a) VEGFA (HR = 4.03, 95\% CI: 3.16-5.15, $P<0.0001)$, (b) SOCS3 (HR = 4.26, 95\% CI: 3.35-5.43, $P<0.0001),(\mathrm{c})$ THBS1 (HR = 2.93, 95\% CI: 2.31-3.72, $P<0.0001)$, (d) SPP1 (HR = 3.80, 95\% CI: 2.98-4.85, $P<0.0001)$, and (e) TGFB2 (HR = 4.64, 95\% CI: 3.64-5.92, $P<0.0001$ ). DE IRGs: differentially expressed immune-related genes; TCGA: The Cancer Genome Atlas; VEGFA: vascular endothelial growth factor A; SOCS3: suppressor of cytokine signaling 3; THBS1: thrombospondin 1; SPP1: secreted phosphoprotein 1; TGFB2: transforming growth factor beta 2 .

TABle 3: Multivariate Cox regression analysis.

\begin{tabular}{lcccrr}
\hline Gene symbol & Description & Coefficient & HR & 95\% CI & $P$ value \\
\hline VEGFA & Vascular endothelial growth factor A & 0.20365 & 1.23 & $1.12-1.34$ & $\mathbf{1 . 0 2 E}-\mathbf{0 5}^{* * *}$ \\
SOCS3 & Suppressor of cytokine signaling 3 & 0.12448 & 1.13 & $1.02-1.25$ & $\mathbf{0 . 0 1 6 5}^{*}$ \\
SPP1 & Secreted phosphoprotein 1 & 0.26231 & 1.30 & $1.20-1.41$ & $\mathbf{1 . 1 0 E}^{*} \mathbf{0 9 ^ { * * * }}$ \\
TGFB2 & Transforming growth factor beta 2 & 0.1293 & 1.14 & $1.02-1.27$ & $\mathbf{0 . 0 1 6 8}^{*}$ \\
\hline
\end{tabular}

HR: hazard ratio; $* * *$ and $*$ shows $P$ value less than 0.01 and 0.05 , respectively; bold texts indicate statistically significant difference. 


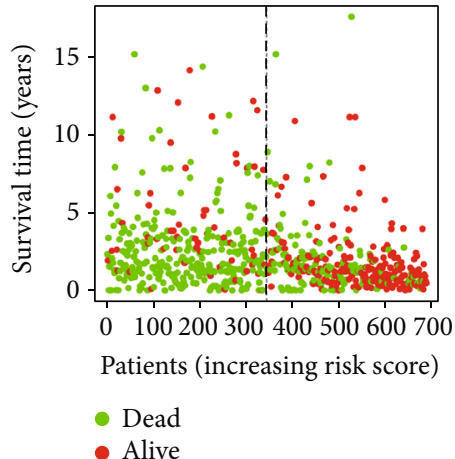

- Alive

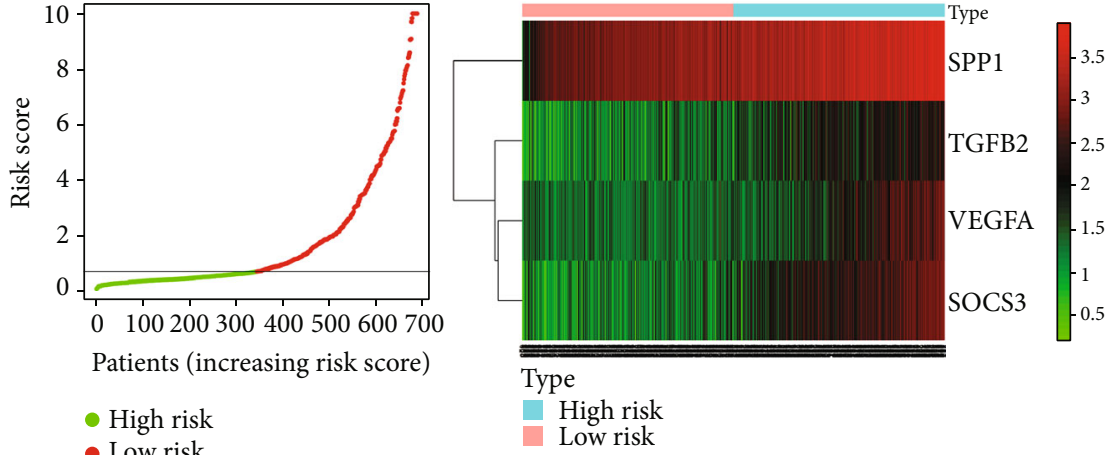

(a)

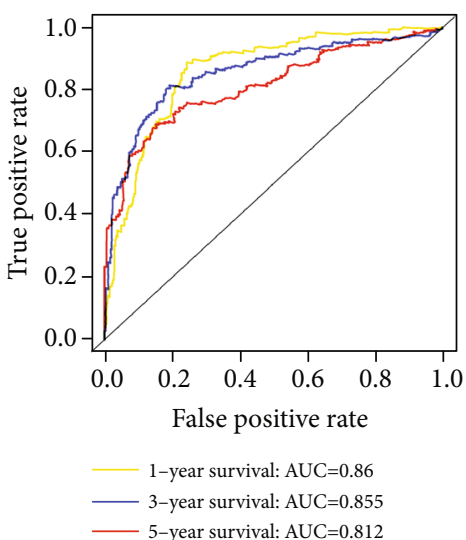

(b)

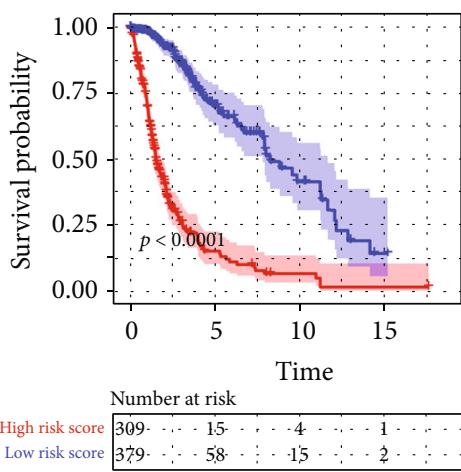

Risk score

+ High risk score

+ Low risk score

(c)

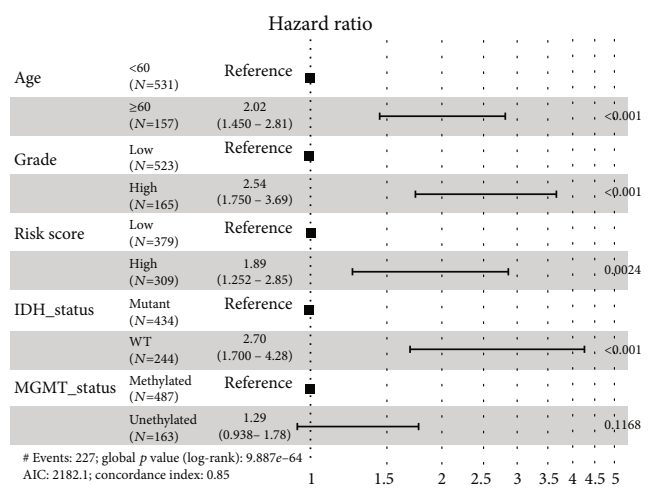

(d)

FIGURE 6: Establishment of an IRRS model in the TCGA cohort. (a) The distribution of risk score in glioma patients based on the IRRS model and survival status of each glioma patient, as well as a heat map of four DE IRG expressions in high- and low-risk groups. (b) ROC curves at 1-year (AUC: 0.86), 3-year (AUC: 0.855), and 5-year (AUC: 0.812) time points for predicting its prognostic performance in patients with glioma. (c) Survival curve reveals high-risk score patients associated with unfavorable outcome, with a 5-year survival rate of roughly $14.3 \%$. (d) Multivariate Cox regression analysis discovers the potentiality of this IRRS model as an independent prognostic factor $(\mathrm{HR}=1.89,95 \% \mathrm{CI}: 1.252-2.85, P=0.0024)$. IRRS: immune-related risk score; TCGA: The Cancer Genome Atlas; DE IRGs: differentially expressed immune-related genes; AUC: area under the curve; OS: overall survival; ROC: receiver operating characteristic.

more than ten genes, which restricted their widespread application.

\section{Discussion}

With the increasing significance of IRG signatures on tumors' prognosis $[29,77,78]$, it is imperative to throw light on their prognostic value for tumors. In our present study, four progression-related DE IRG (including VEGFA, SOCS3, $S P P 1$, and TGFB2 genes)constituting a signature can perform risk stratification for glioma patients and disclosed that patients with high risk seemed to have an approximate $14.3 \%-29.8 \%$ 5-year survival rate. Besides, we speculated that these overexpressed core genes might participate in the 
TABLE 4: The correlations between the risk score and clinicopathological characteristics.

\begin{tabular}{|c|c|c|c|c|c|c|}
\hline \multirow{2}{*}{ Characteristics } & \multicolumn{3}{|c|}{ TCGA cohort $(n=688)$} & \multicolumn{3}{|c|}{ CGGA cohort $(n=422)$} \\
\hline & High risk & Low risk & $P$ value & High risk & Low risk & $P$ value \\
\hline Age & & & $P<0.0001$ & & & $P<0.0001$ \\
\hline$<60$ & 189 & 342 & & 159 & 203 & \\
\hline$\geq 60$ & 120 & 37 & & 46 & 13 & \\
\hline NA & - & - & & 1 & - & \\
\hline Gender & & & 0.8164 & & & 0.4308 \\
\hline Female & 131 & 165 & & 83 & 96 & \\
\hline Male & 178 & 214 & & 123 & 120 & \\
\hline Tumor grade & & & $P<0.0001$ & & & $P<0.0001$ \\
\hline Low & 149 & 374 & & 93 & 189 & \\
\hline High & 160 & 5 & & 113 & 27 & \\
\hline$I D H$ status & & & $P<0.0001$ & & & $P<0.0001$ \\
\hline Mutation & 78 & 356 & & 66 & 142 & \\
\hline Wild-type & 223 & 21 & & 137 & 38 & \\
\hline NA & 8 & 2 & & 3 & 36 & \\
\hline$M G M T$ promoter status & & & $P<0.0001$ & & & 0.2748 \\
\hline Methylation & 148 & 339 & & 90 & 107 & \\
\hline Unmethylation & 124 & 39 & & 76 & 70 & \\
\hline NA & 37 & 1 & & 40 & 39 & \\
\hline
\end{tabular}

TCGA: The Cancer Genome Atlas; CGGA: Chinese Glioma Genome Atlas; IDH: isocitrate dehydrogenase; MGMT: O-6-methylguanine-DNA methyltransferase; NA: not available; bold texts indicate statistically significant difference.

TABLE 5: Univariate and multivariate Cox regression analyses for the IRRS model and clinical traits.

\begin{tabular}{|c|c|c|c|c|c|c|c|c|c|c|c|c|}
\hline \multirow{3}{*}{ Variables } & \multicolumn{6}{|c|}{ Univariate cox analysis } & \multicolumn{6}{|c|}{ Multivariate cox analysis } \\
\hline & \multicolumn{3}{|c|}{ TCGA } & \multicolumn{3}{|c|}{ CGGA } & \multicolumn{3}{|c|}{ TCGA } & \multicolumn{3}{|c|}{ CGGA } \\
\hline & $\begin{array}{c}\text { HR } \\
(95 \% \mathrm{CI})\end{array}$ & $P$ value & $\begin{array}{c}\text { C-index } \\
(95 \% \mathrm{CI})\end{array}$ & $\begin{array}{c}\text { HR } \\
(95 \% \text { CI) }\end{array}$ & $P$ value & $\begin{array}{c}\text { C-index } \\
(95 \% \text { CI })\end{array}$ & $\begin{array}{c}\text { HR } \\
(95 \% \mathrm{CI})\end{array}$ & $\begin{array}{c}P \\
\text { value }\end{array}$ & $\begin{array}{c}\text { C-index } \\
(95 \% \mathrm{CI})\end{array}$ & $\begin{array}{c}\text { HR } \\
(95 \% \mathrm{CI})\end{array}$ & $P$ value & $\begin{array}{c}\text { C-index } \\
(95 \% \mathrm{CI})\end{array}$ \\
\hline Age & $\begin{array}{c}4.89 \\
(3.772- \\
6.33)\end{array}$ & $<0.0001$ & $\begin{array}{c}0.671 \\
(0.640- \\
0.702)\end{array}$ & $\begin{array}{c}3.15 \\
(2.269- \\
4.39)\end{array}$ & $\begin{array}{c}8.25 E \\
-12\end{array}$ & $\begin{array}{c}0.581 \\
(0.554- \\
0.608)\end{array}$ & $\begin{array}{c}2.02 \\
(1.450- \\
2.81)\end{array}$ & $\begin{array}{c}3.20 E \\
-05\end{array}$ & & $\begin{array}{c}1.21 \\
(0.821- \\
1.79)\end{array}$ & 0.3349 & \\
\hline Gender & $\begin{array}{c}1.26 \\
(0.986- \\
1.61)\end{array}$ & 0.0645 & $\begin{array}{c}0.533 \\
(0.500- \\
0.566)\end{array}$ & $\begin{array}{c}1.16 \\
(0.875- \\
1.53)\end{array}$ & 0.309 & $\begin{array}{c}0.506 \\
(0.471- \\
0.541)\end{array}$ & - & - & & - & - & \\
\hline Grade & $\begin{array}{c}9.23 \\
(7.095- \\
12.01)\end{array}$ & $<0.0001$ & $\begin{array}{c}0.736 \\
(0.709- \\
0.763)\end{array}$ & $\begin{array}{c}4.20 \\
(2.851- \\
6.17)\end{array}$ & $\begin{array}{c}3.43 E \\
-13\end{array}$ & $\begin{array}{c}0.638 \\
(0.609- \\
0.667)\end{array}$ & $\begin{array}{c}2.54 \\
(1.750- \\
3.69)\end{array}$ & $\begin{array}{c}9.50 E \\
-07\end{array}$ & & $\begin{array}{c}3.05 \\
(1.916- \\
4.86)\end{array}$ & $2.61 E-06$ & \\
\hline $\begin{array}{l}\text { Risk } \\
\text { score }\end{array}$ & $\begin{array}{c}6.53 \\
(4.960- \\
8.59)\end{array}$ & $<0.0001$ & $\begin{array}{c}0.743 \\
(0.719- \\
0.767)\end{array}$ & $\begin{array}{c}3.85 \\
(2.857- \\
5.19)\end{array}$ & $<0.0001$ & $\begin{array}{c}0.661 \\
(0.630- \\
0.692)\end{array}$ & $\begin{array}{c}1.89 \\
(1.252- \\
2.85)\end{array}$ & $\begin{array}{c}2.40 E \\
-03\end{array}$ & $\begin{array}{c}(0.823- \\
0.871)\end{array}$ & $\begin{array}{c}2.17 \\
(1.493- \\
3.14)\end{array}$ & $4.63 E-05$ & \\
\hline $\begin{array}{l}\text { IDH_ } \\
\text { status }\end{array}$ & $\begin{array}{c}8.76 \\
(6.708- \\
11.43)\end{array}$ & $<0.0001$ & $\begin{array}{c}0.782 \\
(0.758- \\
0.806)\end{array}$ & $\begin{array}{c}4.42 \\
(3.278- \\
5.96)\end{array}$ & $<0.0001$ & $\begin{array}{c}0.699 \\
(0.672- \\
0.726)\end{array}$ & $\begin{array}{c}2.70 \\
(1.700- \\
4.28)\end{array}$ & $\begin{array}{c}2.51 E \\
-05\end{array}$ & & $\begin{array}{c}2.85 \\
(1.966- \\
4.14)\end{array}$ & $3.46 E-08$ & \\
\hline $\begin{array}{l}M G M T_{-} \\
\text {status }\end{array}$ & $\begin{array}{c}3.17 \\
(2.430- \\
4.13)\end{array}$ & $<0.0001$ & $\begin{array}{c}0.654 \\
(0.619- \\
0.689)\end{array}$ & $\begin{array}{c}1.36 \\
(1.009- \\
1.82)\end{array}$ & 0.0434 & $\begin{array}{c}0.528 \\
(0.489- \\
0.567)\end{array}$ & $\begin{array}{c}1.29 \\
(0.938- \\
2.78)\end{array}$ & 0.1168 & & $\begin{array}{c}1.20 \\
(0.884- \\
1.64)\end{array}$ & 0.2389 & \\
\hline
\end{tabular}

HR: hazard ratio; IRRS: immune-related risk score; TCGA: The Cancer Genome Atlas; CGGA: Chinese Glioma Genome Atlas; IDH: isocitrate dehydrogenase; MGMT: O-6-methylguanine-DNA methyltransferase; bold texts indicate statistically significant difference.

dismal prognosis of patients suffering from glioma via the extracellular matrix (ECM) binding's molecular function. ECM functions as a pivotal role in communicating with a variety of cell types, such as fibroblasts, immune cells, endothelial cells, epithelial cells, and pericytes, via all sorts of cell surface receptors, for regulating their functions and behaviors [79].

$V E G F A$, called vascular endothelial growth factor $\mathrm{A}$, belongs to the $P D G F / V E G F$ growth factor family and plays 

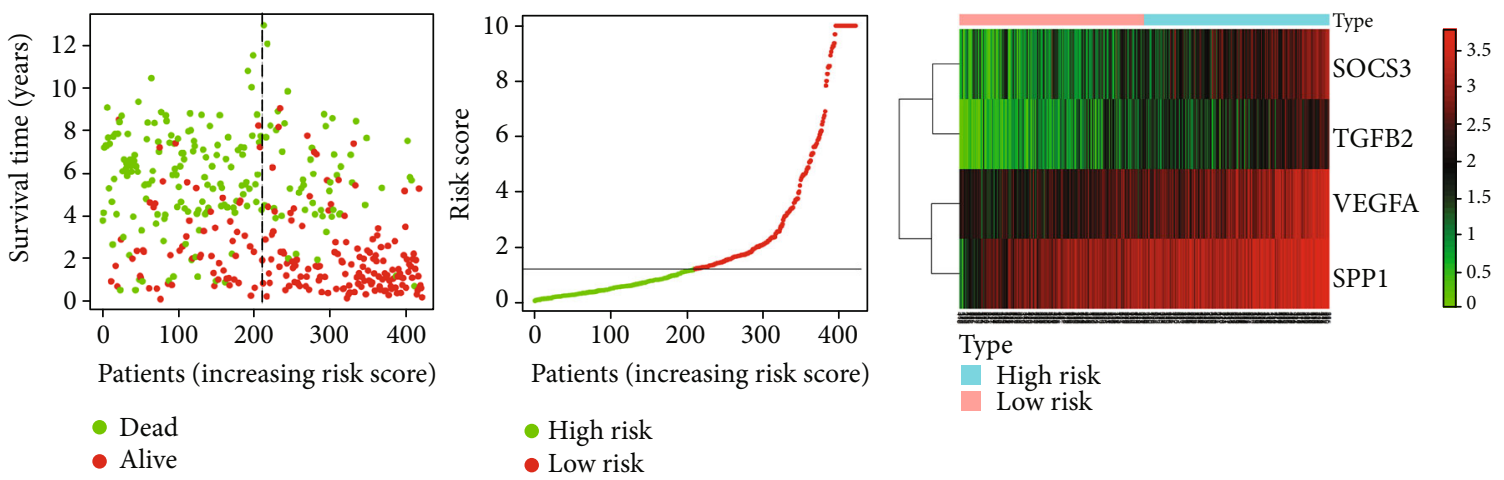

(a)
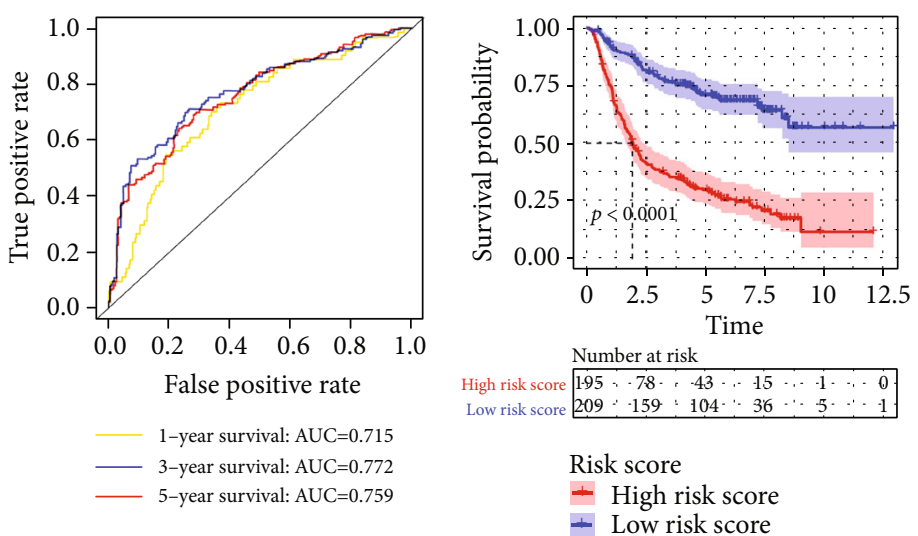

(b)

(c)

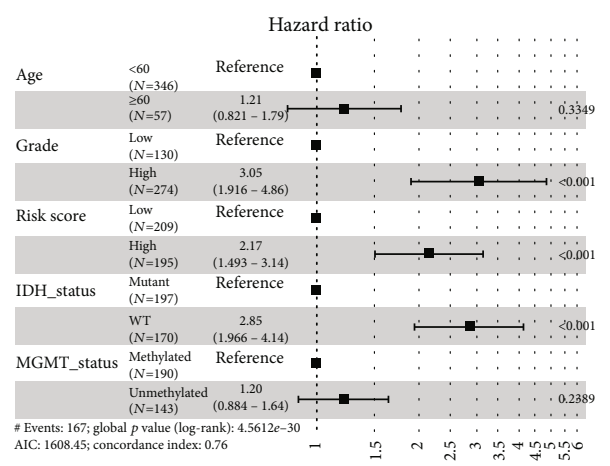

(d)

FIGURE 7: Validation of the IRRS model in the CGGA dataset. (a) The distribution of risk score, survival status of every patient with glioma, and heat map of four DE IRGs. (b) ROC curves of this IRRS prognostic model for evaluating the predictive probability for gliomas' 1-year (AUC: 0.715), 3-year (AUC: 0.772), and 5-year (AUC: 0.759) OS. (c) KM curve indicates glioma patients with high risk has dismal prognosis, with 5-year survival rate of nearly $29.8 \%$. (d) Multivariate Cox regression analysis for the risk score and clinical characteristics denotes a superior prognostic value of the IRRS model for glioma patients ( $\mathrm{HR}=2.17,95 \% \mathrm{CI}: 1.493-3.14, P<0.0001)$. IRRS: immunerelated risk score; CGGA: Chinese Glioma Genome Atlas; DE IRGs: differentially expressed immune-related genes; AUC: area under the curve; OS: overall survival; KM: Kaplan-Meier; ROC: receiver operating characteristic.

an essential part in inducing neovascularization [80] and promoting endothelial cell proliferation and migration. For example, the enzyme for degrading ECM can release active fragments from the matrix substance and activate growth factors, including the heparin-binding epidermal growth factor, insulin-like growth factor, epidermal growth factor, $V E G F$, and fibroblast growth factor-2, not only promoting the growth but also propelling invasion and neovascularization of tumors [81, 82]. Also, VEGFA can act as a crucial regulator of the cancer-immune circulation via generating considerable modifications, inducing immune tolerance and causing tumor immune evasion. Furthermore, soluble proteins coded by the VEGFA gene were involved in the mechanism of inducing angiogenesis and immunosuppressive responses in gastric cancer [83]. In addition, Hatva et al. [84] measured the expression of VEGF in both the normal brain vessel system and glioma cells; then, they concluded that VEGF was manifestly overexpressed in 


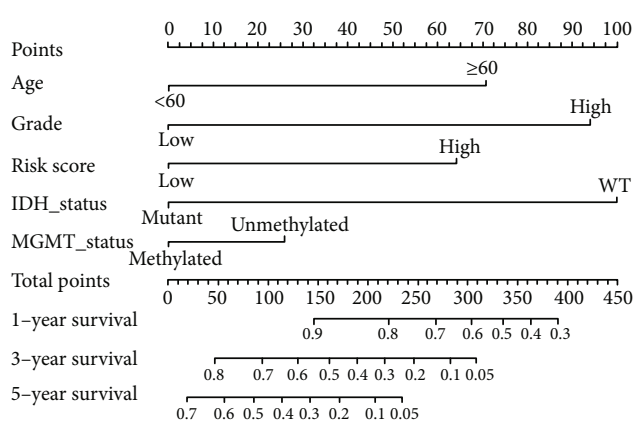

(a)
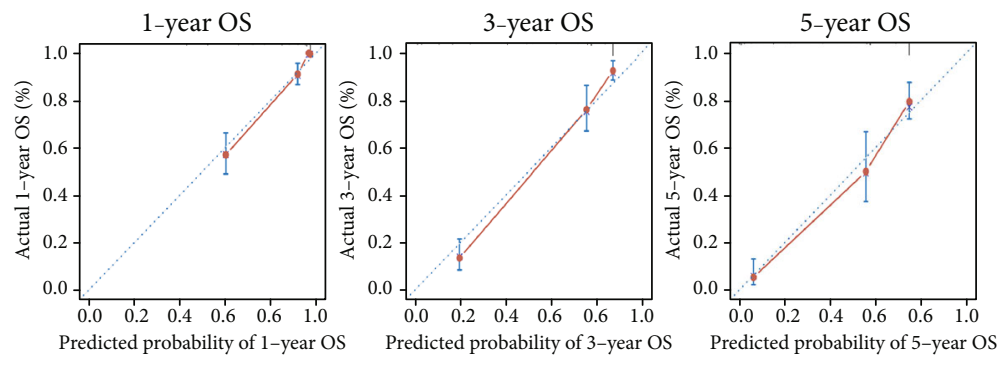

(b)

FIgURE 8: (a) Development of a nomogram and (b) calibration curve analysis. The $x$-axis and $y$-axis separately represent the predicted and actual OS from the nomogram. The diagonal line indicates that the predicted probability is consistent with the actual probability. The solid line and vertical line show the predicted nomogram and 95\% confidence interval, respectively. WT: wild type; OS: overall survival.

malignant glioma cells and their corresponding receptors were induced, which played a cardinal role in the angiogenesis of tumors. Besides, Zhang et al. [85] found that escalation of VEGFA participated in the progression of glioma and lowered patients' OS. Additionally, a high level of VEGFA was relevant to hypoxia, angiogenesis, and immune suppression of the tumor microenvironment (TME) [86], which elaborated the mechanism of tumors' innate resistance to ICIs and uncovered that upregulated VEGFA enhanced the malignancy of tumor cells [87]. Some evidence indicated that a high level of VEGFA had the potentiality of facilitating treatment efficacy based on target therapy. Compared to monotherapy, in the bearing-tumor mice without high expression of VEGFA, combination treatment showed no synergistic effect in antitumor efficacy. Surprisingly, in treatment of tumors with overexpressed VEGFA, antiangiogenesis therapies have transformed the immunosuppressive TME and enhanced the effects of ICIs [87]. Thus, VEGFA can serve as not only a prognostic biomarker for predicting gliomas' prognosis but also a potential immunotherapeutic target in the near future.

SOCS3, suppressor of cytokine signaling 3 , is one of the most potent members of the SOCS family and encodes a group of STAT inhibitors induced by STAT, which contains a motif of the kinase-inhibitory region (KIR) and directly inhibits signal transduction via suppressing the Janus kinase (JAK) catalytic activity $[88,89]$. To our knowledge, suppressive SOCS3 is a negative modulator of cytokine signaling via directly inhibiting JAKs, functioning as a key regulator of the immune system [90]. Besides, in the central nervous system, the expressed process of IFN- $\beta$-induced SOCS3 in astrocytes depended on the activation of STAT3. Destruction of the expression of SOCS3 caused a great deal of inflammatory responses and promoted the migration of microglial and $\mathrm{T}$ cells [91]. Shi et al. [92] revealed that the signaling pathway of ER-JAK2/STAT3/SOCS3 was activated by bisphenol F, which propelled the polarization of macrophages to the proinflammatory M1 subtype. Additionally, McFarland et al. [93] found that myeloid cell population without SOCS3 delayed the growth of intracranial tumors and raised survival rates in the orthotopic glioma-bearing mice. Also, Sheu et al. have found a novel transcriptional mechanism of ECM accumulation induced by high glucose. Specifically, high glucose enhanced SOCS3 expression via activating PI3K and STAT1/3, which produced a multitude of cascade reactions to form the ECM [94]. Therefore, we believe that SOCS3 will be a promising prognostic biomarker of patients with glioma.

$S P P 1$, a secreted phosphoprotein 1, also called osteopontin $(O P N)$, encodes the chemokine-like, calcified ECMassociated proteins. It plays a pivotal role in regulating immune functions and participating in adhesion, remodeling ECM, proliferation, and angiogenesis, as well as metastasis of tumors [95, 96]. Saitoh et al. [97] demonstrated that $O P N$ had a close relation to glioma's malignancy. $O P N$ is one of the most significant components of the ECM, which is involved in the regulation of matrix interactions and cell adhesion. Also, OPN was able to interact with ECM elements, such as collagen, fibronectin, and calcium ion [98]. Besides, Friedmann-Morvinski et al. [99] indicated that silencing of $O P N$ significantly exerted influence on the cell cycle and WNT as well as focal adhesion signaling pathways in GBM patients. At the same time, they have drawn a conclusion that $O P N$ serves a crucial role in cell dedifferentiation during the formation of tumors. As a consequence, inhibition of $O P N$ might be the potential target for the treatment of GBM. Also, OPN was overexpressed in almost $90 \%$ GBM patients, and a high level of OPN was associated with tumor malignancy, where $O P N$ recruited neutrophils and macrophages, inducing tumor cell and leukocyte migration [100]. Similarly, macrophages were recruited by OPN to the site of GBM, implying that OPN acted as a crosstalk between glioma cells and the innate immune system. Thus, OPN could be considered to be an outstanding therapeutic target [101].

TGFB2, transforming growth factor beta 2 , encodes the ligands of the TGF-beta super-family's proteins. These ligands bind a variety of TGF-beta receptors, leading to recruiting SMAD transcription factors, which modulate gene expressions. Chen et al. [102] found that malignant glioma cells were able to regulate some adhesion molecules (such as VCAM-1) via secreting TGF-beta2 and releasing the tumor necrosis factor (TNF) receptor. Of note, Corbet et al. [103] demonstrated that TGF-beta2 was the main 


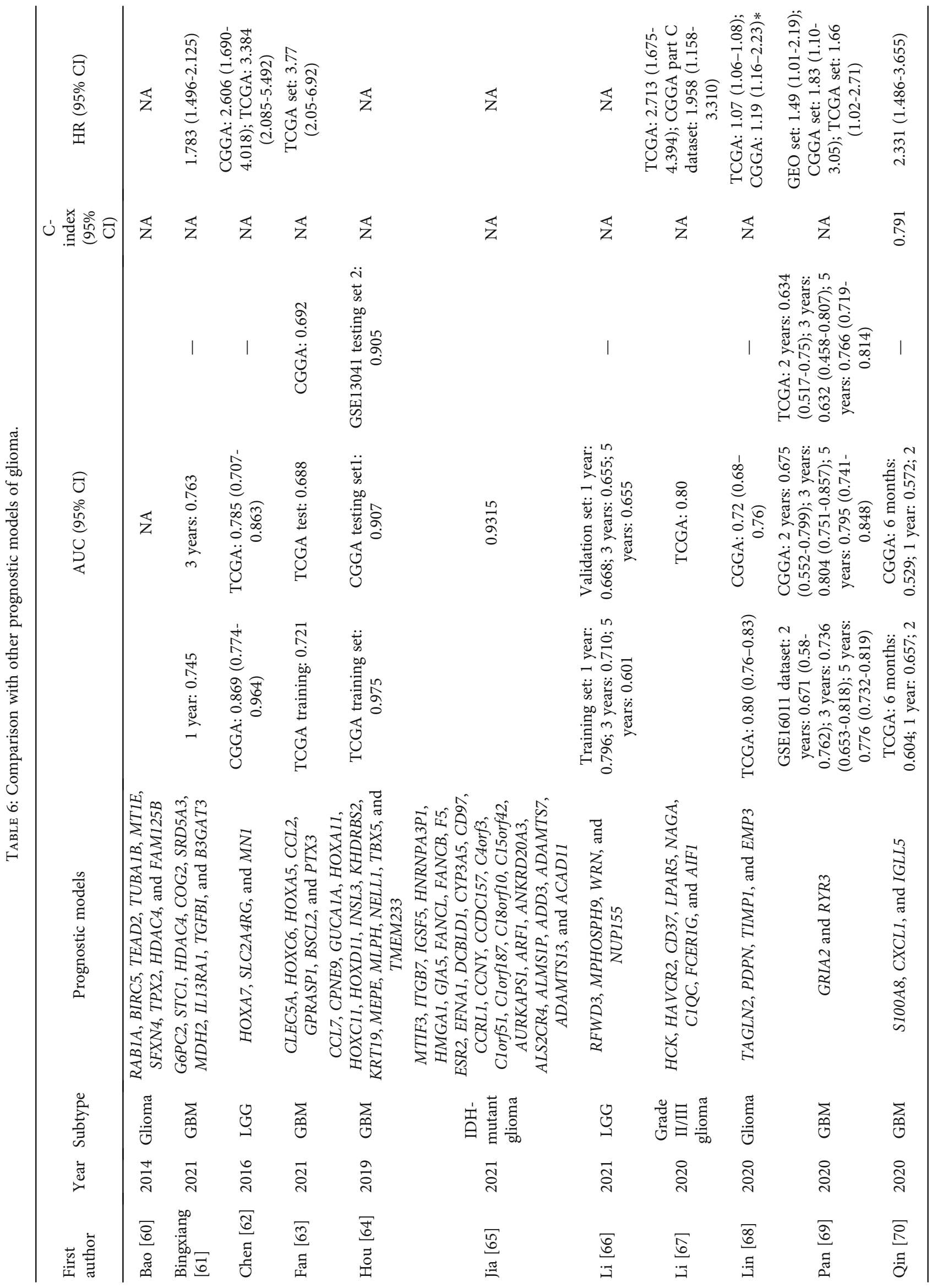




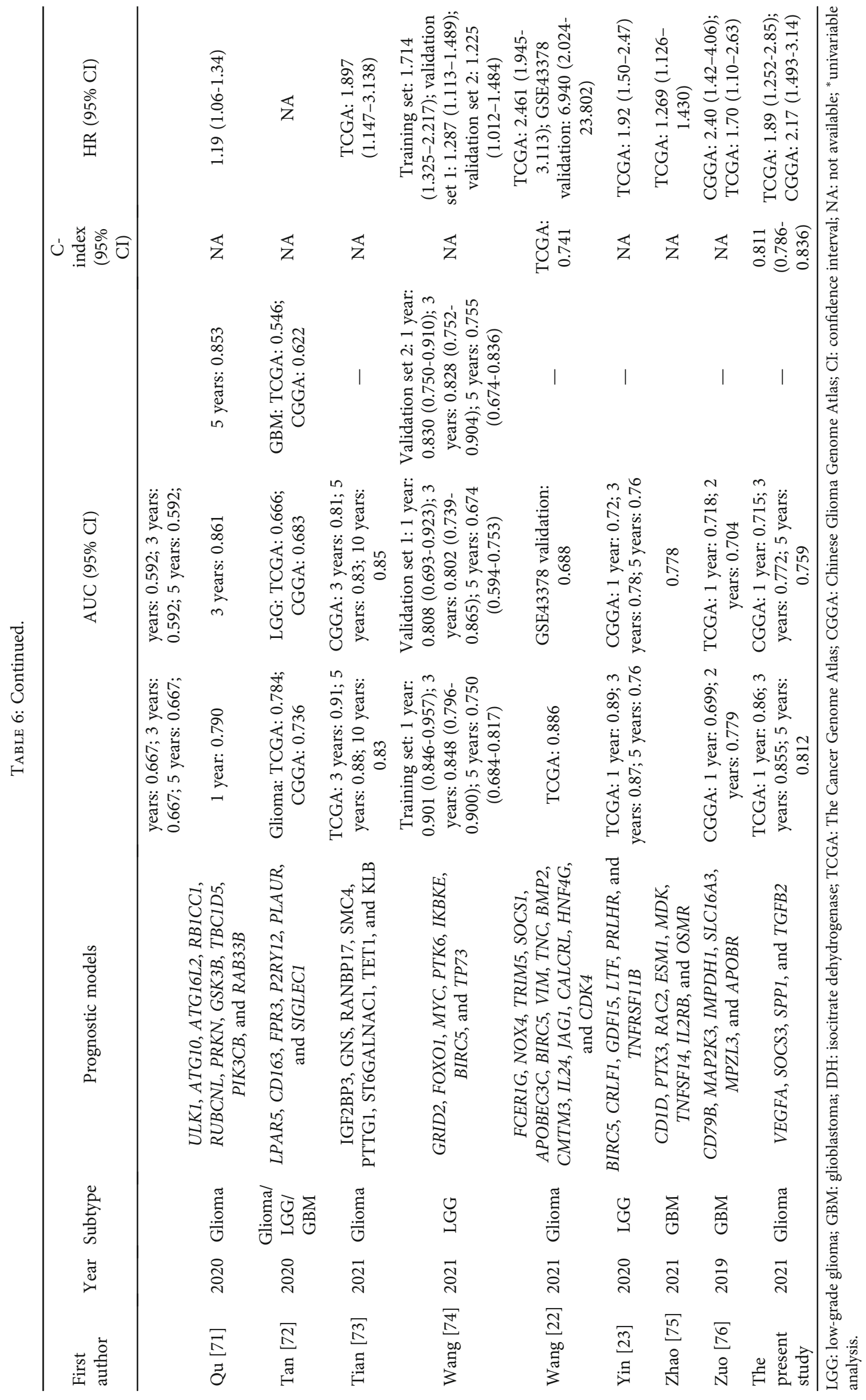


driving force of the reconstruction of lipid metabolism promoted by tumor acidosis and was essential to support energy requirements of cancer cells' invasion. Moreover, they also found that acidosis-induced TGF-beta2 activation promoted partial epithelial-to-mesenchymal transition (EMT) and fatty acid metabolism in various original tumor cells, where the latter supported the acetylation of Smad2. Similarly, TGF-beta2 activated the autophagy of human glioma cell lines via the Smad and non-Smad pathways, facilitating glioma cell invasion, where epithelial-interstitial transformation and metabolic alterations were vital for glioma progression [104]. Additionally, Xiao et al. [105] showed that TGF-beta2 functioned as a key factor influencing immune cell recruitment and infiltration to the gastric tumors' site. Therefore, TGF-beta2 may be regarded as a valuable prognostic biomarker for tumors.

There were some advantages in the present study. First, we adopted the top-ranking 5000 gene expression values based on the MAD algorithm for performing WGCNA, avoiding utilizing DEGs, which was not recommended by the official website. Second, we also removed the batchbatch difference and performed differential expression analysis using the integrated transcriptome analysis based on four GEO datasets, which enabled our results to be more reliable. However, the limitations also need to be given more importance. Given that glioma tissues were difficult to collect, it is challenging to perform experimental validation in gene expressions. Furthermore, it is also rather restricted to construct a model of glioma progression using glioma cell lines for our laboratory. Finally, the specific mechanism and interrelationship of core IRGs in the IRRS model deserve to be further investigated.

\section{Conclusions}

Taken together, in the present study, we established an IRRS prognostic model, composed of VEGFA, SOCS3, SPP1, and TGFB2 core DE IRGs, for risk stratification and survival prediction for glioma patients. Additionally, glioma patients with high risk stratified by this IRRS prognostic model presented a short survival time, which might be attributed to the ECM signal pathway where those genes participate in. However, a multitude of prospective studies and experiments will be needed to verify our findings in the near future.

\section{Data Availability}

The data that support the findings of this study are available from The Cancer Genome Atlas database (https://portal.gdc .cancer.gov/), GEO database (GSE45921, GSE15824, GSE43378, GSE4290, and GPL570, http://www.ncbi.nlm .nih.gov/geo/), Chinese Glioma Genome Atlas (CGGA) database (http://www.cgga.org.cn/), Immunology Database and Analysis Portal (ImmPort) database (https://www .immport.org/shared/home), and cBioPortal for Cancer Genomics database (http://www.cbioportal.org/).

\section{Disclosure}

The funding agencies had no role in the study design, analysis, interpretation of results, decision to publish, or preparation of the manuscript. The content is solely the responsibility of the authors and does not necessarily represent the official views of the funding agency.

\section{Conflicts of Interest}

The authors declare that there is no conflict of interest.

\section{Acknowledgments}

We are appreciative of the researchers who provided the information in the TCGA, CGGA, GEO, ImmPort, and cBioPortal databases. We also gratefully acknowledge Yongcan Guo $\mathrm{PhD}$ for revising the manuscript. This research was supported by the Research Project of Suining Central Hospital (no. 2021y43).

\section{Supplementary Materials}

Supplementary 1. Supplementary Table 1: the genes in the turquoise module.

Supplementary 2. Supplementary Table 2: the DEGs between HGG and LGG samples.

\section{References}

[1] Q. Ostrom, G. Cioff, H. Gittleman et al., "CBTRUS statistical report: primary brain and other central nervous system tumors diagnosed in the United States in 2012-2016," Neuro-Oncology, vol. 21, Supplement 5, pp. v1-v100, 2019.

[2] M. Weller, M. van den Bent, J. C. Tonn et al., "European Association for Neuro-Oncology (EANO) guideline on the diagnosis and treatment of adult astrocytic and oligodendroglial gliomas," Lancet Oncology, vol. 18, no. 6, pp. e315e329, 2017.

[3] D. N. Louis, H. Ohgaki, O. D. Wiestler et al., “The 2007 WHO classification of tumours of the central nervous system," Acta Neuropathologica, vol. 114, no. 2, pp. 97-109, 2007.

[4] S. Fouke, T. Benzinger, D. Gibson, T. C. Ryken, S. N. Kalkanis, and J. J. Olson, "The role of imaging in the management of adults with diffuse low grade glioma," Journal of NeuroOncology, vol. 125, no. 3, pp. 457-479, 2015.

[5] R. Soffietti, B. G. Baumert, L. Bello et al., "Guidelines on management of low-grade gliomas: report of an EFNS-EANO task force," European Journal Of Neurology, vol. 17, no. 9, pp. 1124-1133, 2010.

[6] M. Kiran, A. Chatrath, X. Tang, D. M. Keenan, and A. Dutta, "A prognostic signature for lower grade gliomas based on expression of long non-coding RNAs," Molecular Neurobiology, vol. 56, no. 7, pp. 4786-4798, 2019.

[7] T. Jiang, Y. Mao, W. Ma et al., "CGCG clinical practice guidelines for the management of adult diffuse gliomas," Cancer Letters, vol. 375, no. 2, pp. 263-273, 2016.

[8] R. Stupp, S. Taillibert, A. Kanner et al., "Effect of tumortreating fields plus maintenance temozolomide vs maintenance temozolomide alone on survival in patients with 
glioblastoma: a randomized clinical trial," JAMA, vol. 318, no. 23 , pp. 2306-2316, 2017.

[9] M. Sanmamed and L. Chen, "A paradigm shift in cancer immunotherapy: from enhancement to normalization," Cell, vol. 175, no. 2, pp. 313-326, 2018.

[10] H. Wang, T. Xu, Q. Huang, W. Jin, and J. Chen, "Immunotherapy for malignant glioma: current status and future directions," Trends In Pharmacological Sciences, vol. 41, no. 2, pp. 123-138, 2020.

[11] S. Topalian, C. Drake, and D. Pardoll, "Immune checkpoint blockade: a common denominator approach to cancer therapy," Cancer Cell, vol. 27, no. 4, pp. 450-461, 2015.

[12] T. Hodges, M. Ott, J. Xiu et al., "Mutational burden, immune checkpoint expression, and mismatch repair in glioma: implications for immune checkpoint immunotherapy," Neuro Oncologia, vol. 19, no. 8, pp. 1047-1057, 2017.

[13] A. Omuro, G. Vlahovic, M. Lim et al., "Nivolumab with or without ipilimumab in patients with recurrent glioblastoma: results from exploratory phase I cohorts of CheckMate 143," Neuro-Oncology, vol. 20, no. 5, pp. 674-686, 2018.

[14] D. Reardon, A. Brandes, A. Omuro et al., "Effect of nivolumab vs bevacizumab in patients with recurrent glioblastoma," JAMA Oncology, vol. 6, no. 7, pp. 1003-1010, 2020.

[15] R. Kandimalla, H. Tomihara, J. K. Banwait et al., “A 15-gene immune, stromal, and proliferation gene signature that significantly associates with poor survival in patients with pancreatic ductal adenocarcinoma," Clinical Cancer Research, vol. 26, no. 14, pp. 3641-3648, 2020.

[16] D. Guo, M. Wang, Z. Shen, and J. Zhu, "A new immune signature for survival prediction and immune checkpoint molecules in lung adenocarcinoma," Journal of Translational Medicine, vol. 18, no. 1, p. 123, 2020.

[17] W. Jin, Y. Zhang, Z. Liu, Z. Che, M. Gao, and H. Peng, "Exploration of the molecular characteristics of the tumorimmune interaction and the development of an individualized immune prognostic signature for neuroblastoma," Journal of Cellular Physiology, vol. 236, no. 1, pp. 294-308, 2021.

[18] J. Yang, K. Xie, and C. Li, "Immune-related genes have prognostic significance in head and neck squamous cell carcinoma," Life Sciences, vol. 256, article 117906, 2020.

[19] M. Zhang, X. Wang, X. Chen, Q. Zhang, and J. Hong, "Novel immune-related gene signature for risk stratification and prognosis of survival in lower-grade glioma," Frontiers in Genetics, vol. 11, 2020.

[20] W. Wang, Z. Zhao, F. Yang et al., "An immune-related lncRNA signature for patients with anaplastic gliomas," Journal of Neuro-Oncology, vol. 136, no. 2, pp. 263-271, 2018.

[21] W. Cheng, X. Ren, C. Zhang et al., "Bioinformatic profiling identifies an immune-related risk signature for glioblastoma," Neurology, vol. 86, no. 24, pp. 2226-2234, 2016.

[22] J.-J. Wang, H. Wang, B.-L. Zhu et al., "Development of a prognostic model of glioma based on immune-related genes," Oncology Letters, vol. 21, no. 2, p. 116, 2021.

[23] W. Yin, X. Jiang, J. Tan et al., "Development and validation of a tumor mutation burden-related immune prognostic model for lower-grade glioma," Frontiers in Oncology, vol. 10, 2020.

[24] S. Yang, Y. Wu, Y. Deng et al., "Identification of a prognostic immune signature for cervical cancer to predict survival and response to immune checkpoint inhibitors," OncoImmunology, vol. 8, no. 12, article e1659094, 2019.
[25] J. Zhou, T. Xu, Y. Yan et al., "MicroRNA-326 functions as a tumor suppressor in glioma by targeting the nin one binding protein (NOB1)," PLoS One, vol. 8, no. 7, article e68469, p. e68469, 2013.

[26] M. Grzmil, P. Morin, M. Lino et al., "MAP kinase-interacting kinase 1 regulates SMAD2-dependent TGF- $\beta$ signaling pathway in human glioblastoma," Cancer Research, vol. 71, no. 6, pp. 2392-2402, 2011.

[27] A. Kawaguchi, N. Yajima, N. Tsuchiya et al., "Gene expression signature-based prognostic risk score in patients with glioblastoma," CANCER SCIENCE, vol. 104, no. 9, pp. 1205-1210, 2013.

[28] J. Sun, M. A. Hui, Q. Su et al., "Neuronal and glioma-derived stem cell factor induces angiogenesis within the brain," CANCER CELL, vol. 9, no. 4, pp. 287-300, 2006.

[29] L. Sun, Z. Zhang, Y. Yao, W. Y. Li, and J. Gu, "Analysis of expression differences of immune genes in non-small cell lung cancer based on TCGA and ImmPort data sets and the application of a prognostic model," Annals of translational medicine, vol. 8, no. 8, p. 550, 2020.

[30] Y. Wang, T. Qian, G. You et al., "Localizing seizuresusceptible brain regions associated with low-grade gliomas using voxel-based lesion-symptom mapping," NEUROONCOLOGY, vol. 17, no. 2, pp. 282-288, 2015.

[31] X. Liu, Y. Li, Z. Qian et al., "A radiomic signature as a noninvasive predictor of progression-free survival in patients with lower-grade gliomas," Neuroimage Clinical, vol. 20, pp. 1070-1077, 2018.

[32] J. H. Liu, S. Y. Li, J. Y. Liang et al., "ITLNI identified by comprehensive bioinformatic analysis as a hub candidate biological target in human epithelial ovarian cancer," Cancer Management and Research, vol. 11, pp. 2379-2392, 2019.

[33] J. Tang, M. Lu, Q. Cui et al., "Overexpression of ASPM, CDC20, and TTK confer a poorer prognosis in breast cancer identified by gene co-expression network analysis," Frontiers in Oncology, vol. 9, p. 310, 2019.

[34] Z. Liu, M. Li, X. Fang et al., "Identification of surrogate prognostic biomarkers for allergic asthma in nasal epithelial brushing samples by WGCNA," JOURNAL OF CELLULAR Biochemistry, vol. 120, no. 4, pp. 5137-5150, 2019.

[35] A. Maertens, V. Tran, A. Kleensang, and T. Hartung, "Weighted gene correlation network analysis (WGCNA) reveals novel transcription factors associated with bisphenol A dose-response," Frontiers in Genetics, vol. 9, p. 508, 2018.

[36] L. Zhang, W. Kang, X. Lu, S. Ma, L. Dong, and B. Zou, "Weighted gene co-expression network analysis and connectivity map identifies lovastatin as a treatment option of gastric cancer by inhibiting HDAC2," Gene, vol. 681, pp. 15-25, 2019.

[37] P. Langfelder, B. Zhang, and S. Horvath, "Defining clusters from a hierarchical cluster tree: the Dynamic Tree Cut package for R," BIOINFORMATICS, vol. 24, no. 5, pp. 719-720, 2008.

[38] M. Ding, F. Li, B. Wang, G. Chi, and H. Liu, "A comprehensive analysis of WGCNA and serum metabolomics manifests the lung cancer-associated disordered glucose metabolism," JOURNAL OF CELLULAR BIOCHEMISTRY, vol. 120, no. 6, pp. 10855-10863, 2019.

[39] M. Mason, G. Fan, K. Plath, Q. Zhou, and S. Horvath, "Signed weighted gene co-expression network analysis of transcriptional regulation in murine embryonic stem cells," $B M C$ GENOMICS, vol. 10, no. 1, p. 327, 2009. 
[40] J. Zhang, J. Fan, C. Zhou, and Y. Qi, “miR-363-5p as potential prognostic marker for hepatocellular carcinoma indicated by weighted co-expression network analysis of miRNAs and mRNA," BMC GASTROENTEROLOGY, vol. 17, no. 1, p. 81, 2017.

[41] T. Zhang, J. Guo, J. Gu et al., "KIAA0101 is a novel transcriptional target of FoxM1 and is involved in the regulation of hepatocellular carcinoma microvascular invasion by regulating epithelial-mesenchymal transition," Journal of Cancer, vol. 10, no. 15, pp. 3501-3516, 2019.

[42] S. Yepes, R. López, R. E. Andrade, P. A. Rodriguez-Urrego, L. López-Kleine, and M. M. Torres, "Co-expressed miRNAs in gastric adenocarcinoma," GENOMICS, vol. 108, no. 2, pp. 93-101, 2016.

[43] L. Gautier, L. Cope, B. M. Bolstad, and R. A. Irizarry, "affyanalysis of Affymetrix GeneChip data at the probe level," BIOINFORMATICS, vol. 20, no. 3, pp. 307-315, 2004.

[44] B. M. Bolstad, R. A. Irizarry, M. Astrand, and T. P. Speed, “A comparison of normalization methods for high density oligonucleotide array data based on variance and bias," BIOINFORMATICS, vol. 19, no. 2, pp. 185-193, 2003.

[45] Z. Zhao, N. Timofeev, S. W. Hartley et al., "Imputation of missing genotypes: an empirical evaluation of IMPUTE," BMC GENETICS, vol. 9, no. 1, p. 85, 2008.

[46] J. T. Leek, W. E. Johnson, H. S. Parker, A. E. Jaffe, and J. D. Storey, "The sva package for removing batch effects and other unwanted variation in high-throughput experiments," BIOINFORMATICS, vol. 28, no. 6, pp. 882-883, 2012.

[47] G. Yu, L. G. Wang, Y. Han, and Q. Y. He, "clusterProfiler: an $\mathrm{R}$ package for comparing biological themes among gene clusters," OMICS-A JOURNAL OF INTEGRATIVE BIOLOGY, vol. 16, no. 5, pp. 284-287, 2012.

[48] J. A. Blake, J. Chan, R. Kishore, P. W. Sternberg, and Y. Li, "Gene Ontology Consortium: going forward," NUCLEIC ACIDS RESEARCH, vol. 43, no. D1, pp. D1049-D1056, 2015.

[49] M. Kanehisa, "Molecular network analysis of diseases and drugs in KEGG," Methods Molecular Biology, vol. 939, no. 10, pp. 263-275, 2013.

[50] D. Szklarczyk, J. H. Morris, H. Cook et al., "The STRING database in 2017: quality-controlled protein-protein association networks, made broadly accessible," NUCLEIC ACIDS RESEARCH, vol. 45, no. D1, pp. D362-D368, 2017.

[51] P. Shannon, A. Markiel, O. Ozier et al., "Cytoscape: a software environment for integrated models of biomolecular interaction networks," GENOME RESEARCH, vol. 13, no. 11, pp. 2498-2504, 2003.

[52] G. Scardoni, G. Tosadori, M. Faizan, F. Spoto, F. Fabbri, and C. Laudanna, "Biological network analysis with CentiScaPe: centralities and experimental dataset integration," F1000Research, vol. 3, p. 139, 2014.

[53] M. Liu, Z. Xu, Z. du et al., "The identification of key genes and pathways in glioma by bioinformatics analysis," Journal of Immunology Research, vol. 2017, Article ID 1278081, 9 pages, 2017.

[54] W. Walter, F. Sánchez-Cabo, and M. Ricote, "GOplot: an R package for visually combining expression data with functional analysis," BIOINFORMATICS, vol. 31 , no. 17 , pp. 2912-2914, 2015.

[55] H. Akaike, "A new look at the statistical model identification," IEEE Transactions on Automatic Control, vol. 19, no. 6, pp. 716-723, 1974.
[56] P. Heagerty, T. Lumley, and M. Pepe, "Time-dependent ROC curves for censored survival data and a diagnostic marker," BIOMETRICS, vol. 56, no. 2, pp. 337-344, 2000.

[57] G. Yan and T. Greene, "Investigating the effects of ties on measures of concordance," STATISTICS IN Medicine, vol. 27, no. 21, pp. 4190-4206, 2008.

[58] Y. She, X. Kong, Y. Ge et al., "Immune-related gene signature for predicting the prognosis of head and neck squamous cell carcinoma," Cancer Cell International, vol. 20, no. 1, p. 22, 2020.

[59] R. Huang, Z. Chen, W. Li, C. Fan, and J. Liu, "Immune system-associated genes increase malignant progression and can be used to predict clinical outcome in patients with hepatocellular carcinoma," INTERNATIONAL JOURNAL OF ONCOLOGY, vol. 56, no. 5, pp. 1199-1211, 2020.

[60] Z. S. Bao, M. Y. Li, J. Y. Wang et al., "Prognostic value of a nine-gene signature in glioma patients based on mRNA expression profiling," CNS Neuroscience \& Therapeutics, vol. 20, no. 2, pp. 112-118, 2014.

[61] X. Bingxiang, W. Panxing, F. Lu, Y. Xiuyou, and D. Chao, “A prognostic model for brain glioma patients based on 9 signature glycolytic genes," Biomed Research International, vol. 2021, Article ID 6680066, 15 pages, 2021.

[62] B. Chen, T. Liang, P. Yang et al., "Classifying lower grade glioma cases according to whole genome gene expression," Oncotarget, vol. 7, no. 45, article 12188, pp. 74031-74042, 2016.

[63] F. Fan, H. Zhang, Z. Dai et al., "A comprehensive prognostic signature for glioblastoma patients based on transcriptomics and single cell sequencing," Cellular Oncology, vol. 44, no. 4, pp. 917-935, 2021.

[64] Z. Hou, J. Yang, H. Wang, D. Liu, and H. Zhang, "A potential prognostic gene signature for predicting survival for glioblastoma patients," Biomed Research International, vol. 2019, Article ID 9506461, 9 pages, 2019.

[65] Y. Jia, W. Yang, B. Tang, Q. Feng, and Z. Dong, "Hub gene identification and prognostic model construction for isocitrate dehydrogenase mutation in glioma," Translational Oncology, vol. 14, no. 1, article 100979, 2021.

[66] X. Li and Y. Meng, "Construction of a SUMOylation regulator-based prognostic model in low-grade glioma," Journal Of Cellular And Molecular Medicine, vol. 25, no. 12, pp. 5434-5442, 2021.

[67] Y. Li, G. Deng, H. Zhang et al., "Weighted gene correlation network analysis identifies microenvironment-related genes signature as prognostic candidate for grade II/III glioma," Aging (Albany NY), vol. 12, no. 21, article 104075, pp. 22122-22138, 2020.

[68] S. Lin, H. Xu, A. Zhang et al., "Prognosis analysis and validation of $\mathrm{m} 6 \mathrm{~A}$ signature and tumor immune microenvironment in glioma," Frontiers in Oncology, vol. 10, article 541401, 2020.

[69] Y. Pan, J. H. Zhang, L. Zhao et al., "A robust two-gene signature for glioblastoma survival prediction," JOURNAL OF CELLULAR BIOCHEMISTRY, vol. 121, no. 7, pp. 35933605, 2020.

[70] Z. Qin, X. Zhang, Z. Chen, and N. Liu, "Establishment and validation of an immune-based prognostic score model in glioblastoma," INTERNATIONAL IMMUNOPHARMACOLOGY, vol. 85, article 106636, 2020.

[71] S. Qu, S. Liu, W. Qiu, J. Liu, and H. Wang, "Screening of autophagy genes as prognostic indicators for glioma 
patients," American Journal of Translational Research, vol. 12, no. 9, pp. 5320-5331, 2020.

[72] Y. Q. Tan, Y. T. Li, T. F. Yan et al., "Six immune associated genes construct prognostic model evaluate low-grade glioma," Frontiers in Immunology, vol. 11, article 606164, 2020.

[73] R. Tian, Y. Li, Q. Liu, and M. Shu, "Identification and validation of an immune-associated RNA-binding proteins signature to predict clinical outcomes and therapeutic responses in glioma patients," Cancers (Basel), vol. 13, no. 7, p. 1730, 2021.

[74] C. Wang, J. Qiu, S. Chen et al., "Prognostic model and nomogram construction based on autophagy signatures in lower grade glioma," JOURNAL OF CELLULAR PHYSIOLOGY, vol. 236, no. 1, pp. 235-248, 2021.

[75] B. Zhao, Y. Wang, Y. Wang et al., "Systematic identification, development, and validation of prognostic biomarkers involving the tumor-immune microenvironment for glioblastoma," JOURNAL OF CELLULAR Physiology, vol. 236, no. 1, pp. 507-522, 2021.

[76] S. Zuo, X. Zhang, and L. Wang, "A RNA sequencing-based six-gene signature for survival prediction in patients with glioblastoma," Scientific reports, vol. 9, no. 1, p. 2615, 2019.

[77] A. Ling, A. Löfgren-Burström, P. Larsson et al., "TAP1 downregulation elicits immune escape and poor prognosis in colorectal cancer," Onco Immunology, vol. 6, no. 11, article e1356143, 2017.

[78] G. Ding, L. Zhou, T. Shen, and L. Cao, "IFN- $\gamma$ induces the upregulation of RFXAP via inhibition of miR-212-3p in pancreatic cancer cells: a novel mechanism for IFN- $\gamma$ response," Oncology Letters, vol. 15, no. 3, pp. 3760-3765, 2018.

[79] A. D. Theocharis, D. Manou, and N. K. Karamanos, "The extracellular matrix as a multitasking player in disease," The FEBS journal, vol. 286, no. 15, pp. 2830-2869, 2019.

[80] I. Chaudhry, D. O'Donovan, P. Brenchley, H. Reid, and I. S. D. Roberts, "Vascular endothelial growth factor expression correlates with tumour grade and vascularity in gliomas," HISTOPATHOLOGY, vol. 39, no. 4, pp. 409-415, 2001.

[81] N. K. Karamanos, A. D. Theocharis, T. Neill, and R. V. Iozzo, "Matrix modeling and remodeling: a biological interplay regulating tissue homeostasis and diseases," MATRIX BIOLOGY, vol. 75-76, pp. 1-11, 2019.

[82] V. P. Ferrer, V. Moura Neto, and R. Mentlein, "Glioma infiltration and extracellular matrix: key players and modulators," GLIA, vol. 66, no. 8, pp. 1542-1565, 2018.

[83] M. Refolo, C. Lotesoriere, C. Messa, M. G. Caruso, and R. D'Alessandro, "Integrated immune gene expression signature and molecular classification in gastric cancer: new insights," Journal of leukocyte biology, vol. 108, no. 2, pp. 633-646, 2020.

[84] E. Hatva, A. Kaipainen, P. Mentula, and K. Alitalo, "Expression of endothelial cell-specific receptor tyrosine kinases and growth factors in human brain tumors," AMERICAN JOURNAL OF PATHOLOGY, vol. 146, no. 2, pp. 368-378, 1995.

[85] S. Zhang, K. Leung, C. McCrudden, and H. Kwok, "The prognostic significance of combining VEGFA, FLT1 and KDR mRNA expressions in brain tumors," Journal of Cancer, vol. 6, no. 9, pp. 812-818, 2015.

[86] D. Gabrilovich, S. Ostrand-Rosenberg, and V. Bronte, "Coordinated regulation of myeloid cells by tumours," NATURE REVIEWS IMMUNOLOGY, vol. 12, no. 4, pp. 253-268, 2012.
[87] Q. Wang, J. Gao, W. Di, and X. Wu, “Anti-angiogenesis therapy overcomes the innate resistance to PD-1/PD-L1 blockade in VEGFA-overexpressed mouse tumor models," Cancer Immunology, Immunotherapy, vol. 69, no. 9, pp. 1781-1799, 2020.

[88] A. Sasaki, H. Yasukawa, A. Suzuki et al., "Cytokine-inducible $\mathrm{SH} 2$ protein-3 (CIS3/SOCS3) inhibits Janus tyrosine kinase by binding through the $\mathrm{N}$-terminal kinase inhibitory region as well as SH2 domain," GENES TO CELLS, vol. 4, no. 6, pp. 339-351, 1999.

[89] H. Yasukawa, H. Misawa, H. Sakamoto et al., "The JAKbinding protein $\mathrm{JAB}$ inhibits Janus tyrosine kinase activity through binding in the activation loop," EMBO Journal, vol. 18, no. 5, pp. 1309-1320, 1999.

[90] N. Kershaw, J. Murphy, N. Liau et al., "SOCS3 binds specific receptor-JAK complexes to control cytokine signaling by direct kinase inhibition," NATURE STRUCTURAL \& MOLECULAR BIOLOGY, vol. 20, no. 4, pp. 469-476, 2013.

[91] H. Qin, S. Niyongere, S. Lee, B. J. Baker, and E. N. Benveniste, "Expression and functional significance of SOCS-1 and SOCS-3 in astrocytes," JOURNAL OF Immunology, vol. 181, no. 5, pp. 3167-3176, 2008.

[92] M. Shi, Z. Lin, L. Ye et al., "Estrogen receptor-regulated SOCS3 modulation via JAK2/STAT3 pathway is involved in BPF-induced M1 polarization of macrophages," TOXICOLOGY, vol. 433-434, article 152404, pp. 433-434, 2020.

[93] B. McFarland, M. Marks, A. Rowse et al., "Loss of SOCS3 in myeloid cells prolongs survival in a syngeneic model of glioma," Oncotarget, vol. 7, no. 15, pp. 20621-20635, 2016.

[94] M. L. Sheu, C. C. Shen, J. R. Jheng, and C. K. Chiang, "Activation of PI3K in response to high glucose leads to regulation of SOCS- 3 and STAT1/3 signals and induction of glomerular mesangial extracellular matrix formation," Oncotarget, vol. 8, no. 10, pp. 16925-16938, 2017.

[95] H. Rangaswami, A. Bulbule, and G. Kundu, "Osteopontin: role in cell signaling and cancer progression," TRENDS IN CELL BIOLOGY, vol. 16, no. 2, pp. 79-87, 2006.

[96] P. Anborgh, J. Mutrie, A. Tuck, and A. Chambers, "Role of the metastasis-promoting protein osteopontin in the tumour microenvironment," JOURNAL OF CELLULAR AND Molecular Medicine, vol. 14, no. 8, pp. 2037-2044, 2010.

[97] Y. Saitoh, J. Kuratsu, H. Takeshima, S. Yamamoto, and Y. Ushio, "Expression of osteopontin in human glioma. Its correlation with the malignancy," LABORATORY INVESTIGATION, vol. 72, no. 1, pp. 55-63, 1995.

[98] C. M. Giachelli and S. Steitz, "Osteopontin: a versatile regulator of inflammation and biomineralization," MATRIX BIOL$O G Y$, vol. 19, no. 7, pp. 615-622, 2000.

[99] D. Friedmann-Morvinski, V. Bhargava, S. Gupta, I. M. Verma, and S. Subramaniam, "Identification of therapeutic targets for glioblastoma by network analysis," ONCOGENE, vol. 35, no. 5, pp. 608-620, 2016.

[100] P. Sreekanthreddy, H. Srinivasan, D. M. Kumar et al., "Identification of potential serum biomarkers of glioblastoma: serum osteopontin levels correlate with poor prognosis," Cancer Epidemiology, Biomarkers \& Prevention, vol. 19, pp. 1409-1422, 2010.

[101] J. Wei, A. Marisetty, B. Schrand et al., “Osteopontin mediates glioblastoma-associated macrophage infiltration and is a potential therapeutic target," JOURNAL OF CLINICAL INVESTIGATION, vol. 129, no. 1, pp. 137-149, 2019. 
[102] T. Chen, D. Hinton, V. Yong, and F. Hofman, "TGF-B2 and soluble p55 TNFR modulate VCAM-1 expression in glioma cells and brain derived endothelial cells," JOURNAL OF NEUROIMMUNOLOGY, vol. 73, no. 1-2, pp. 155-161, 1997.

[103] C. Corbet, E. Bastien, J. P. S. de Jesus et al., “TGF $\beta 2$-induced formation of lipid droplets supports acidosis-driven EMT and the metastatic spreading of cancer cells," Nature Communications, vol. 11, no. 1, p. 454, 2020.

[104] C. Zhang, X. Zhang, R. Xu et al., "TGF- $\beta 2$ initiates autophagy via Smad and non-Smad pathway to promote glioma cells' invasion," Journal of Experimental \& Clinical Cancer Research, vol. 36, no. 1, p. 162, 2017.

[105] Z. Xiao, L. Hu, L. Yang et al., “TGF $\beta 2$ is a prognostic-related biomarker and correlated with immune infiltrates in gastric cancer," JOURNAL OF CELLULAR AND MOLECULAR MEDICINE, vol. 24, no. 13, pp. 7151-7162, 2020. 